\title{
Double Earthquakes Classification and Seismic Precursors
}

\author{
Giulio Riga', Paolo Balocchi ${ }^{2}$ \\ ${ }^{1}$ Geologist, Independent Researcher, Lamezia Terme, Italy \\ ${ }^{2}$ Geologist, Independent Researcher, Modena, Italy \\ Email: giulio.riga@tin.it
}

How to cite this paper: Riga, G. and $\mathrm{Ba}$ locchi, P. (2018) Double Earthquakes Classification and Seismic Precursors. Open Journal of Earthquake Research, 7, 1-27. https://doi.org/10.4236/ojer.2018.71001

Received: December 8, 2017

Accepted: February 5, 2018

Published: February 8, 2018

Copyright (c) 2018 by authors and Scientific Research Publishing Inc. This work is licensed under the Creative Commons Attribution International License (CC BY 4.0).

http://creativecommons.org/licenses/by/4.0/

\begin{abstract}
In this paper, we examine both the sequence and organisation of major shallow earthquakes occurred in various areas of the world from 1904 to 2017. We aim to describe their major features and how they are connected with foreshocks and aftershocks immediately close in time and space. Examining magnitude value's fluctuations over time, we see that they form a basic pattern, consisting of three maxima, one of which is central, and two or more events preceding and following it, whose magnitude, in some cases, may be comparable. The retrospective analysis of earthquakes' patterns of high comparable magnitude has allowed their classification along with the development of some statistically significant relationships between epicentral distance and magnitude difference and between time interval and delay among maxima as well as the identification of activation signals predicting their occurrence. The pattern we identified in seismic sequences analysis, in relation to minor shocks-generated activation signals' positions may be used to obtain useful information for the evolutionary study of seismic sequences and for predicting double and multiple earthquakes. The graphic analysis procedure applied to the pattern enables us to know the period of seismic sequence's greatest hazard after a strong earthquake.
\end{abstract}

\section{Keywords}

Double Earthquakes, Microsequence, Foreshock, Mainshock, Pattern, Seismicity Fluctuation Prediction

\section{Introduction}

Moderate and strong earthquakes may be preceded and followed by events of comparable magnitude forming a pattern only slightly different from the typical 
foreshocks-mainshock-aftershocks sequence (FMA).

The term Doublet Earthquakes (DEQ) is related to two seismic events showing similar magnitude and localisation values, and occurring after a short delay [1] [2]. DEQ are especially frequent in seismic areas featuring large asperities [3] and may occur along the same fault or a different fault. They may happen a few seconds or minutes after the first shock, or even weeks or months later.

Previously performed studies classify as DEQ two earthquakes whose magnitude difference does not exceed 0.2 units, spatial separation is less than $100 \mathrm{~km}$ and time separation is a few years [3] [4].

DEQ's trigger mechanism is still not clear, but a possible explanation for their occurrence is that the first shock increases the static stress in the crusted adjacent volumes, which show different conditions, resistance and asperities [5]-[10]. In fact, asperities' fracture along a fault plane is a heterogeneous phenomenon, where the breaking of one of them gives rise to tensions along adjacent asperities.

In areas featuring large asperities having similar thickness, increases in stress are high and trigger the breaking of adjacent asperities, thus producing a sequence of two distinct earthquakes although with similar magnitude [3].

Asperities distribution is a simple pattern explaining the occurrence features of large earthquakes in many subduction areas [11].

Alternatively, the triggering of the second earthquake along the second fault may result from breaking propagation up to the end of the first fault [12] [13]. From statistical point of view, it has been shown that FMAs and DEQ are caused by the same physical process, consisting in a single activation mechanism [14].

DEQ occur across the world and unquestionably are a significant phenomenon related to the evaluation of the seismic hazard after a big event.

Their impact on buildings and infrastructures damaged during the first event may be greater during the second event and poses a danger to rescue teams after the first earthquake as well.

Using the FMA scheme, in this study we implemented a graphical approach to analyse strong individual earthquakes (EQ) and DEQ, obtain information on seismic sequence development over time, predict the magnitude of the second DEQ event and define activation signals before their occurrence.

The information obtained can be used to define seismic sequence's highest hazards after a strong EQ and plan all actions required for persons and structures' safety to limit the damage.

\section{Methodology}

Observations of numerous seismic sequences have highlighted that inside them it is possible to detect patterns that may be described as warning signals for the most energetic shock. Theoretically, the seismic patterns that develop before an energetic event contain a series of information that allows, in some cases, to predict the shock's energy, days to weeks early by their temporal development. 
The analysis of historical earthquakes' seismic sequences and, especially, of seismic patterns consisting of magnitude values' fluctuations, identifies three types of shocks:

Foreshocks: premonitory shock;

Mainshock: primary shock—often single but sometimes multiple;

Aftershocks:

The preparation process that leads to the nucleation of large earthquakes usually is longer compared to smaller earthquakes': this is also supported by the branched structure that in stronger earthquakes shows greater hierarchisation [15].

We also noted that some EQs are sometimes preceded by minor premonitory shocks, called foreshocks, which create specific short and medium-term patterns characterized by upward trend and higher development rate. Foreshocks may occur individually or in groups, while the time of occurrence between the last foreshocks and the main shock varies from a few hours to a few months depending on their order within the sequence [16].

Usually, first order foreshocks are followed by an aftershocks sequence that delays the main event occurrence, while in second order foreshocks, aftershocks are almost absent. The main shock (mainshock) has greater magnitude and is always followed by a series of lower magnitude shocks (aftershocks), lasting from a few weeks to some years, depending on mainshock magnitude. Aftershocks usually form a downward sequence, consisting of subsequent energy accumulation and release phases [15] whose intensity is lower compared to main event's.

In principle, FMA sequence shows that an earthquake may generate other earthquakes that tend to cluster over time to form a premonitory pattern according to predetermined rules, such as TT-7S [17]. In its simplified form, this pattern consists of a central maximum, mainshock (M) and two secondary side maxima, foreshock (F) and aftershock (A).

By observing magnitude values fluctuations within the seismic sequence, we note that during the energy accumulation phase, pattern TT-7S it is repeated over time with greater frequency, while in the energy release phase it appears as a strong fluctuation of magnitude values.

FMA pattern develops over a more or less short period and may feature some variations including multiple events of the same type that may be close in time and space (DEQ). Figure 1 shows FMA patterns as dashed, red color rectangles and some variations that are formed during seismic sequence development. Green circles indicate the maximum F, while red and yellow circles, indicate the maximum $M$ and $A$, respectively. As can be seen, maximum $M$ is the most energetic in FMA pattern, while maximum $\mathrm{F}$ and $\mathrm{A}$ have a smaller magnitude.

In Figure 2, the dashed red line joining the minimum points in FMA pattern is the transition line that provides information on the pattern completion and dynamic magnitude values of the most energetic aftershocks [18]. In the event 


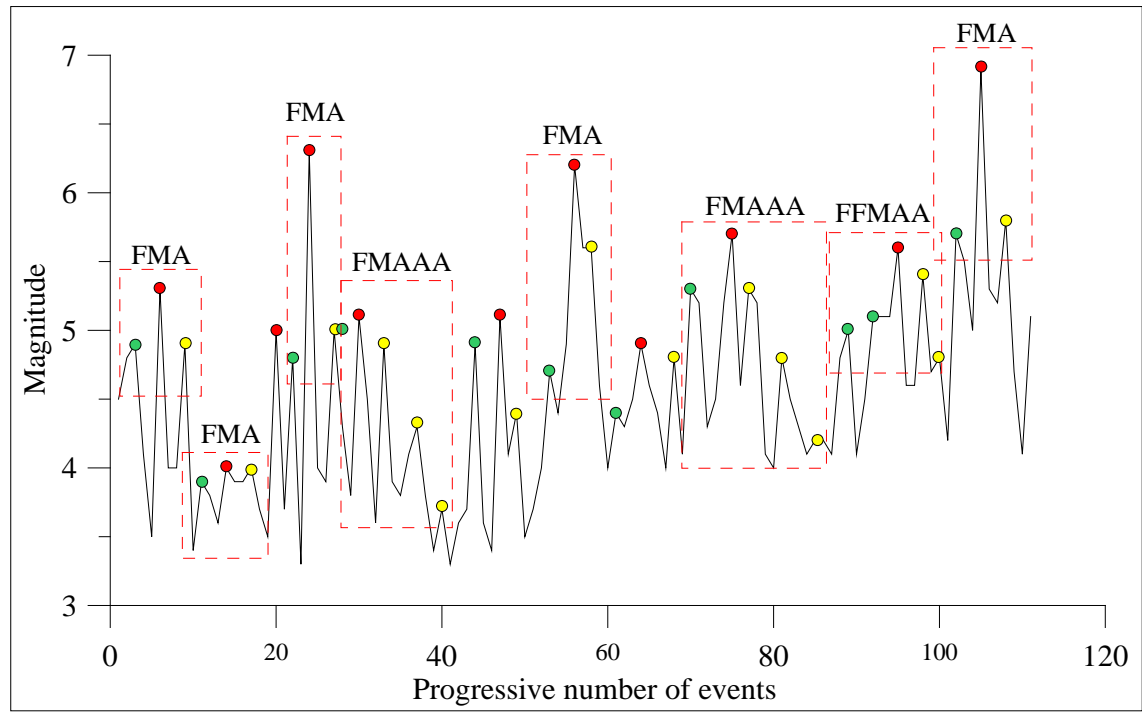

Figure 1. FMA pattern fluctuations and its possible variations.

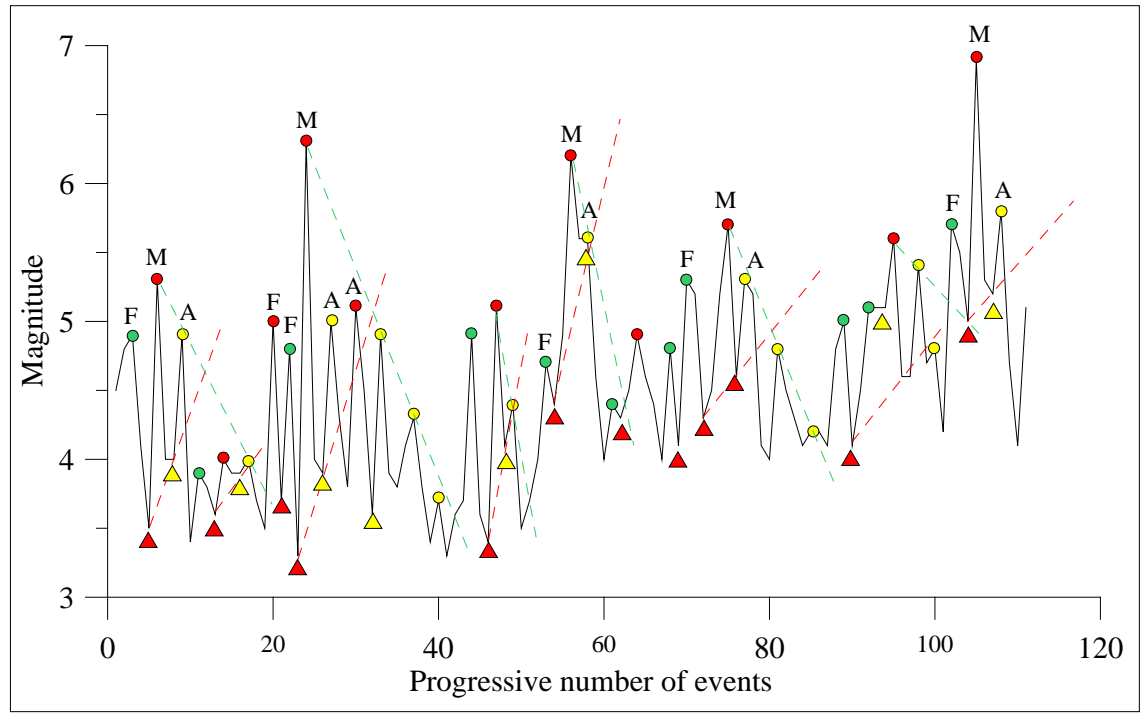

Figure 2. The dashed red line indicates the transition line; the dotted green line shows the aftershock phase's trend line, red and yellow triangles stand for activation signals.

that the second minimum's magnitude is lower than the first's, we should draw the transition line from the first to third maximum (A), and update it if greater magnitude maxima are formed (Figure 7(a) and Figure 7(d)).

FMA pattern organisation in space, in time and magnitude probably is a single triggering process as well as a simple tool to distinguish mainshock from other shocks.

It is assumed that foreshocks' magnitude values depend on past seismicity and are placed above the trend line in the aftershocks phase (green dashed line).

As a rule, in this analytical graphic approach, aftershocks are arranged according to decreasing minimum and maximum values (downward trend), while foreshocks are arranged based on increasing maximum and minimum values 
(upward trend) which are observed before major earthquakes.

During FMA formation, it is possible to observe the following development characteristics:

a) An increase in the number of shocks before the first maximum formation (F), followed by an aftershocks phase consisting of fewer shocks forming a first minimum;

b) The formation of the second maximum (M) characterised by a rapid energy release, and followed, in some cases, by a fast aftershocks phase characterised by shocks of decreasing magnitude forming a second minimum;

c) The formation of a third maximum (A) that often cannot exceed the second maximum's magnitude value $(\mathrm{M})$, but it is frequently above the first maximum's magnitude value $(\mathrm{F})$;

d) The most energetic phase ends when magnitude values fall below the transition line and, during the subsequent rise, fail to cross it, thus signifying the end of the increasing maximum and minimum formation as well as FMA pattern completion;

e) If it is inclined in the ongoing trend's direction, the transition line has greater importance in FMA pattern formation. The higher the inclination, the larger the third maximum's magnitude $(\mathrm{A})$;

f) The activation signal preceding the second maximum (M) coincides with microsequence's fourth shock DB-3SE [15] [17] or with the minimum value in the aftershocks phase following the first maximum $(\mathrm{F})$;

g) The activation signal preceding the third maximum (A) coincides with the minimum formed after the second maximum $(\mathrm{M})$.

In some sequences, a precursor pattern forms before the second maximum (M) formation in FMA pattern, which consists of seven shocks at least with trend opposite to FMA pattern (Figure 3 ). This pattern is called Reverse-FMA

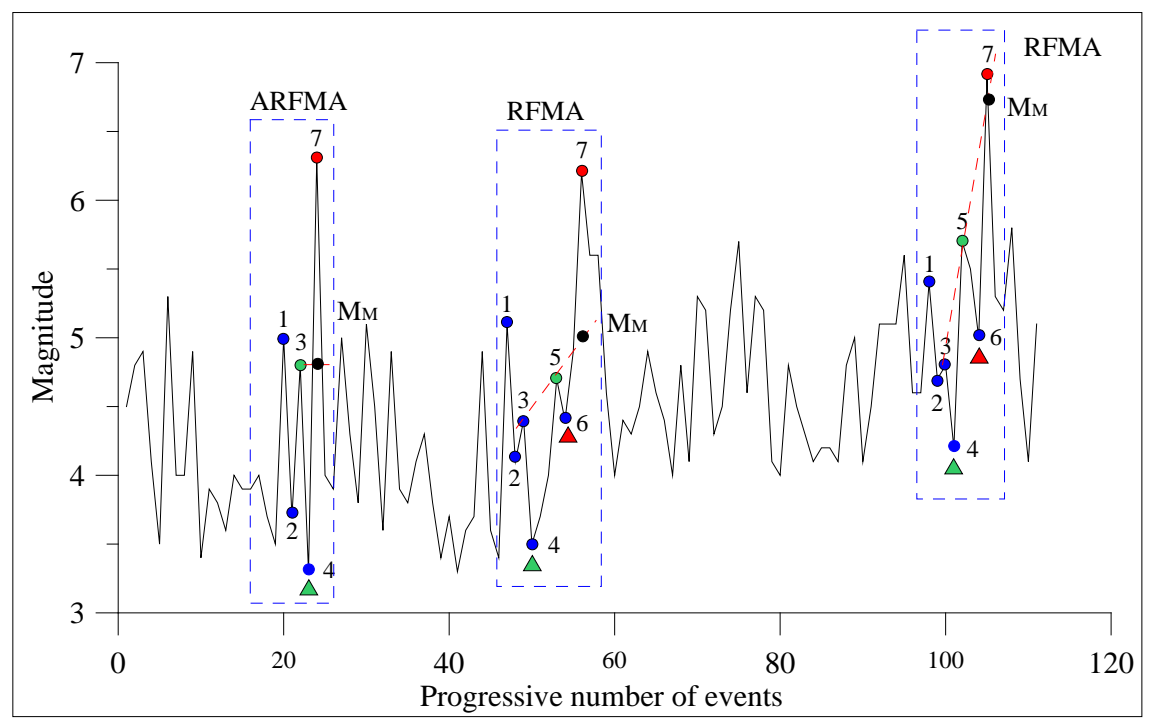

Figure 3. The dashed red line shows the transition line while green and red triangles indicate the activation signals. 
(RFMA), is specular to FMA pattern and usually is formed at the end of magnitude values' upward trend.

Going into details, RFMA pattern development entails the formation of three minimum (points 2, 4,6) and four maximum points $(1,3,5,7$ ), where the second and sixth minimum and the third and the fifth maximum show increasing magnitude. In this pattern, if magnitude values do not form the third minimum (point 6), we have the early formation of a fourth maximum (M). In this case, the transition line is a horizontal line starting from the first maximum (point 3): the pattern is called early RFMA (PRFMA).

This pattern features some minor differences compared to standard FMA patterns:

1) Magnitude values of points 5 and 6 are usually higher than first and second minimum's (points 2 and 4);

2) The transition line joining maximum points 3 and 5 (dashed red line) provides information on minimum mainshock's magnitude value (MM) and on completion of the most energetic phase when magnitude values drop below it;

3) The first activation signal coincides with the second minimum (green triangle), while the second signal coincides with $t$ pattern's third minimum (red triangle).

The third maximum magnitude values' range in FMA pattern can be calculated drawing from the first maximum (F) the line parallel to the transition line (dashed red line) by considering the distance between the transition and the parallel line (red arrow) as the expected magnitude values' dynamic range (Figure $4(\mathrm{a}))$.

In principle, after reaching a minimum in the aftershocks phase (point 2), magnitude values temporarily go back to about $50 \%$ of the second maximum amplitude (M), calculated based on the transition line, before starting descending again.

In some cases, they increase over $50 \%$ by forming two EQ having similar magnitude (DEQ) while sometimes the magnitude values exceed several times the transition line before definitely dropping and forming multiple earthquakes (MEQ).

After the magnitude values' drop below the transition line, the minimum magnitude value can be calculated by measuring the distance between mainshock and transition line (green arrow) and drawing the latter from the breaking point downwards, perpendicularly to the transition line (Figure 4(b)).

Often the minimum target is reached and exceeded during aftershocks phase. In this case, it is possible to estimate a second minimum target by projecting from magnitude value's transit point below the transition line, in addition to the second maximum (green arrow) and the first maximum (red arrow) amplitude.

The preliminary range of fourth maximum magnitude values in RFMA (point 7) (Figure 4(c)) can be calculated by drawing from the first maximum (point 1) a line parallel to the transition line and considering the distance between transition 


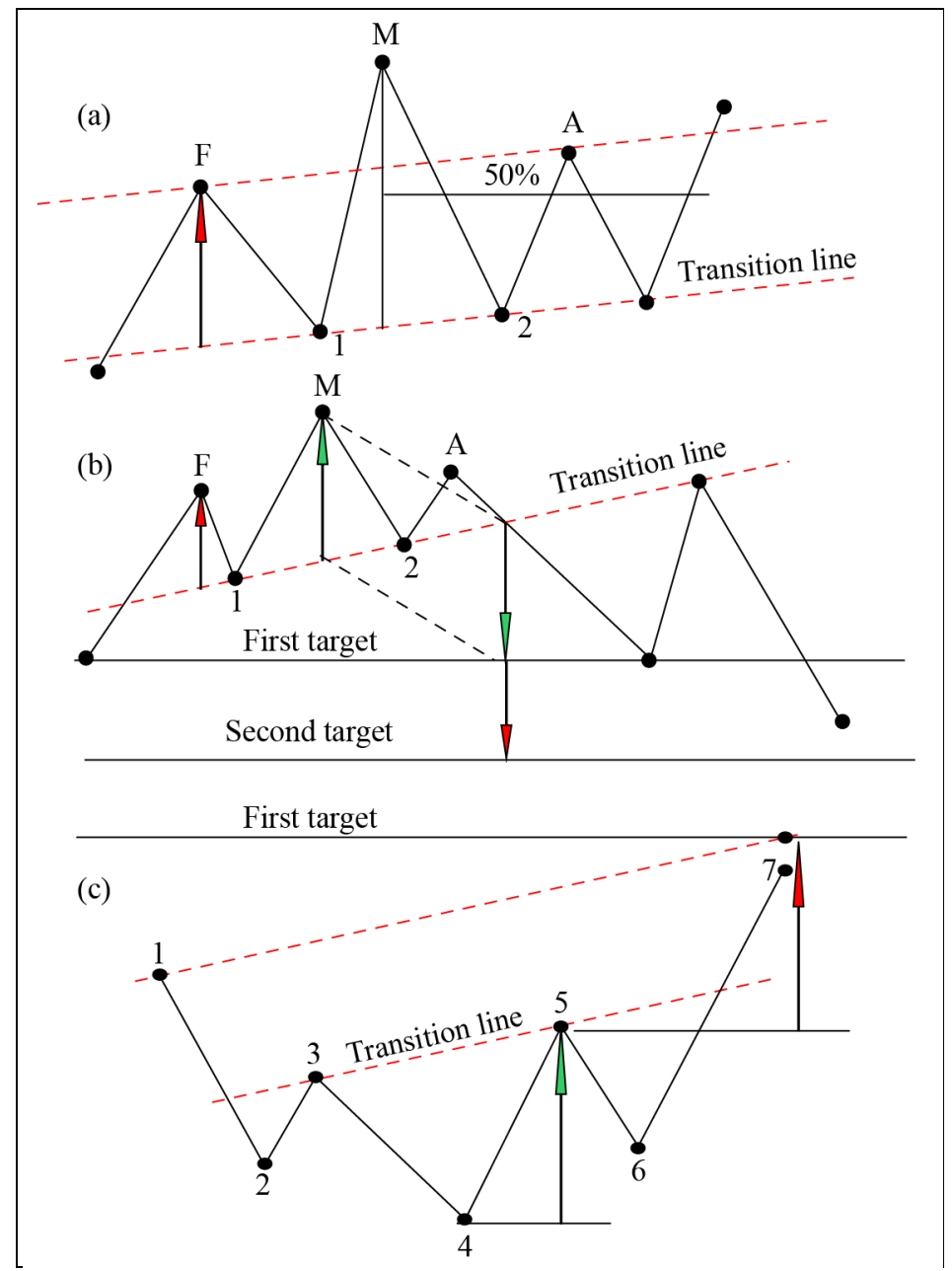

Figure 4. FMA and RFMA patterns and magnitude targets calculation.

and parallel line as the expected magnitude values' dynamic range. The fourth maximum magnitude value may also be computed by measuring the distance between the second minimum (point 4) and the transition line (green arrow) and projecting the latter from the second maximum (point 5) upwards (red arrow).

\section{Results}

In order to classify DEQ occurred in various parts of the world, we analysed several seismic sequences using the catalogs of the National Institute of Geophysics and Volcanology (INGV) [19] and the U.S. Geological Survey (USGS) [20]. For each sequence, we examined individual and double earthquakes as well as changes in foreshocks, mainshock and aftershocks-related activities.

Based on seismicity fluctuation, we identified two earthquakes categories:

1) individual earthquakes;

2) double and multiple earthquakes.

In the first category, the largest shock is easily identifiable because of its greater magnitude compared to other earthquakes in the sequence. 
Individual earthquakes include the following typologies:

(A) "Progressive Earthquakes" (Figure 5(a)) where a strong earthquake is preceded by one or more foreshocks of various order, whose epicenter sometimes is close to mainshock's [20];

(B) "Flash Earthquakes" that are not preceded by foreshocks (Figure 5(b)).

Figure 6 shows FMA patterns (green, red and yellow circles) related to eight EQs occurred in Italy from 1997 to 2016 along with the transition line (red), activation signals (red and yellow triangles) and dynamic magnitude target (black circles) of the third maximum estimated based on the line parallel to the transition line (green).

Table 1 reports datasets' spatial coverage (latitude and longitude), depths and magnitude values' range used, and Table 2 displays the information regarding the shocks between the first $(\mathrm{F})$ and the second maximum $(\mathrm{M})$ in FMA pattern.

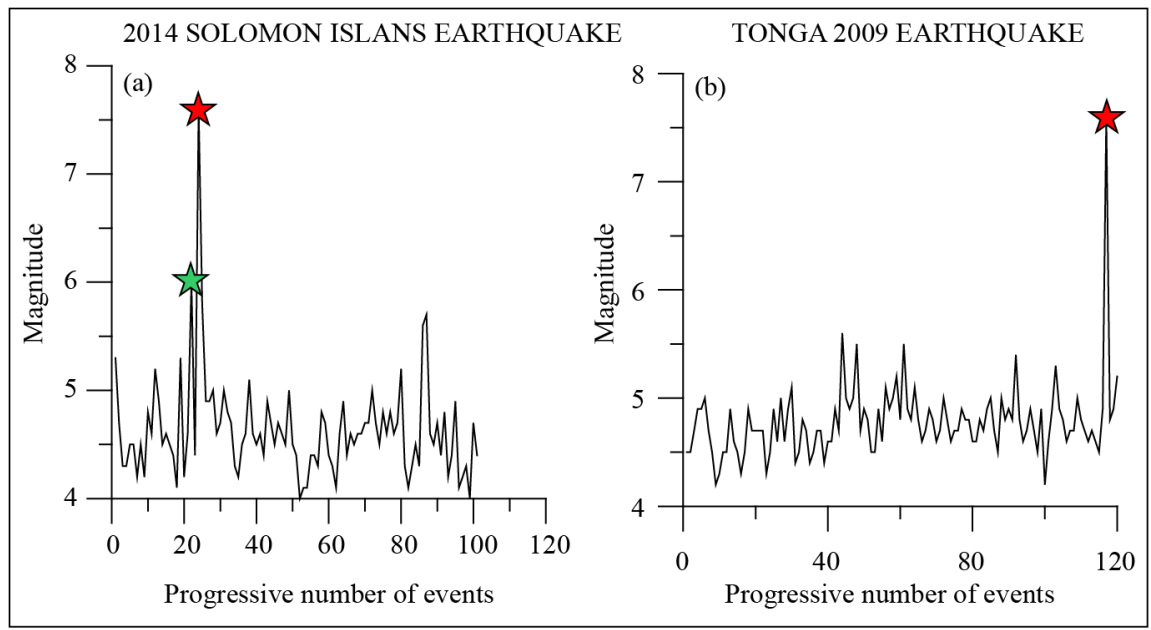

Figure 5. Types of individual earthquake, (a) Progressive earthquakes, (b) Flash earthquake. The red star indicates the mainshock, while the green star shows the foreshock.

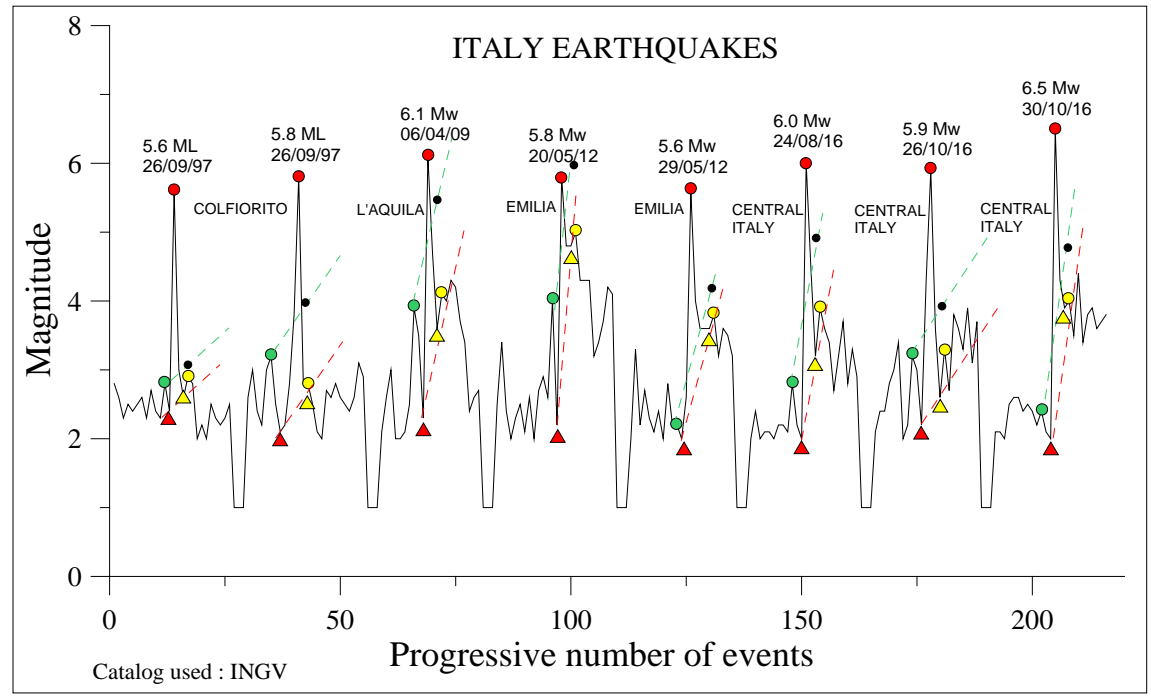

Figure 6. FMA patterns of EQs occurred in Italy from 1997 to 2016. 
Table 1. Earthquakes search parameters in INGV catalog.

\begin{tabular}{|c|c|c|c|c|c|}
\hline No & EARTHQUAKE & LATITUDE & LONGITUDE & DEPTH & MAGNITUDE \\
\hline 1 & Colfiorito & $44 \mathrm{~N}-42.7 \mathrm{~N}$ & $14.2 \mathrm{E}-11.3 \mathrm{E}$ & $0-50$ & $2.0-7.0$ \\
\hline 2 & L'Aquila & $43.2 \mathrm{~N}-41.5 \mathrm{~N}$ & $14.2 \mathrm{E}-12.5 \mathrm{E}$ & $0-50$ & $2.0-7.0$ \\
\hline 3 & Emilia & $45.2 \mathrm{~N}-44.7 \mathrm{~N}$ & $11.7 \mathrm{E}-10.5 \mathrm{E}$ & $0-50$ & $2.0-7.0$ \\
\hline 4 & Central Italy & $43.4 \mathrm{~N}-42.4 \mathrm{~N}$ & $13.7 \mathrm{E}-12.6 \mathrm{E}$ & $0-50$ & $2.0-7.0$ \\
\hline
\end{tabular}

Table 2. Time delays and distance of earthquakes occurred between the first maximum (F) and second maximum (M).

\begin{tabular}{|c|c|c|c|c|c|c|c|c|c|}
\hline No & Earthquakes & Date Event & Lat & Long & $\mathbf{M}$ & $\begin{array}{l}\text { Date Mainshock } \\
\text { (M) }\end{array}$ & $\mathbf{M}$ & $\begin{array}{l}\text { Time Delays } \\
\text { (ddhh:mm) }\end{array}$ & $\begin{array}{c}\text { Distance } \\
(\mathrm{km})\end{array}$ \\
\hline 1 & Colfiorito (F) & 23/09/1997 & 43.030 & 12.902 & $2.8 \mathrm{Md}$ & 26/09/1997 & $5.6 \mathrm{ML}$ & 02 05:07 & 1.60 \\
\hline 2 & Colfiorito (S) & $25 / 09 / 1997$ & 43.031 & 12.878 & $2.4 \mathrm{Md}$ & 26/09/1997 & $5.6 \mathrm{ML}$ & 00 05:04 & 3.19 \\
\hline 3 & Colfiorito (F) & $26 / 09 / 1997$ & 42.996 & 12.966 & $3.2 \mathrm{Md}$ & 26/09/1997 & $5.8 \mathrm{ML}$ & 00 01:10 & 4.50 \\
\hline 4 & Colfiorito & $26 / 09 / 1997$ & 43.028 & 12.867 & $2.5 \mathrm{Md}$ & 26/09/1997 & $5.8 \mathrm{ML}$ & 00 01:00 & 4.92 \\
\hline 5 & Colfiorito (S) & 26/09/1997 & 43.030 & 12.886 & $2.1 \mathrm{Md}$ & 26/09/1997 & $5.8 \mathrm{ML}$ & $0000: 26$ & 3.32 \\
\hline 6 & Colfiorito & $26 / 09 / 1997$ & 43.020 & 12.822 & $2.2 \mathrm{Md}$ & 26/09/1997 & $5.8 \mathrm{ML}$ & $0000: 21$ & 8.47 \\
\hline 7 & Colfiorito & 26/09/1997 & 43.036 & 12.905 & $2.8 \mathrm{Md}$ & 26/09/1997 & $5.8 \mathrm{ML}$ & $0000: 16$ & 2.17 \\
\hline 8 & Colfiorito (F) & $26 / 09 / 1997$ & 43.021 & 12.901 & $3.8 \mathrm{Md}$ & $26 / 09 / 1997$ & $5.8 \mathrm{ML}$ & 00 00:07 & 2.06 \\
\hline 9 & Colfiorito & $26 / 09 / 1997$ & 43.018 & 12.913 & $5.6 \mathrm{ML}$ & 26/09/1997 & $5.8 \mathrm{ML}$ & 00 09:07 & 1.24 \\
\hline 10 & L'Aquila (F) & 05/04/2009 & 42.325 & 13.382 & $3.9 \mathrm{Mw}$ & 06/04/2009 & $6.1 \mathrm{Mw}$ & $0004: 44$ & 1.90 \\
\hline 11 & L'Aquila & 05/04/2009 & 42.329 & 13.385 & $3.5 \mathrm{Mw}$ & $06 / 04 / 2009$ & $6.1 \mathrm{Mw}$ & 00 02:53 & 1.50 \\
\hline 12 & L’Aquila (S) & 05/04/2009 & 42.959 & 13.600 & $2.3 \mathrm{ML}$ & 06/04/2009 & $6.1 \mathrm{Mw}$ & $0002: 36$ & 70.93 \\
\hline 13 & Emilia (F) & $19 / 05 / 2012$ & 44.911 & 11.247 & $4.0 \mathrm{Mw}$ & $20 / 05 / 2012$ & $5.8 \mathrm{Mw}$ & 00 02:50 & 2.14 \\
\hline 14 & Emilia (S) & $19 / 05 / 2012$ & 44.903 & 11.293 & $2.2 \mathrm{ML}$ & $20 / 05 / 2012$ & $5.8 \mathrm{Mw}$ & $00 \quad 02: 21$ & 2.41 \\
\hline 15 & Emilia (S) & $29 / 05 / 2012$ & 44.807 & 11.394 & $2.0 \mathrm{ML}$ & $29 / 05 / 2012$ & $5.6 \mathrm{Mw}$ & $0002: 03$ & 26.16 \\
\hline 16 & Emilia & $29 / 05 / 2012$ & 44.870 & 11.353 & $2.6 \mathrm{ML}$ & $29 / 05 / 2012$ & $5.6 \mathrm{Mw}$ & $0001: 26$ & 22.84 \\
\hline 17 & Central Italy (F) & $16 / 08 / 2016$ & 43.383 & 12.771 & $2.8 \mathrm{ML}$ & $24 / 08 / 2016$ & $6.0 \mathrm{Mw}$ & $0713: 57$ & 84.96 \\
\hline 18 & Central Italy & $16 / 08 / 2016$ & 42.832 & 13.025 & $2.2 \mathrm{ML}$ & $24 / 08 / 2016$ & $6.0 \mathrm{Mw}$ & 07 05:54 & 22.65 \\
\hline 19 & Central Italy (S) & $23 / 08 / 2016$ & 43.057 & 12.988 & $2.0 \mathrm{ML}$ & $24 / 08 / 2016$ & $6.0 \mathrm{Mw}$ & $0006: 48$ & 44.67 \\
\hline 20 & Central Italy (F) & $26 / 10 / 2016$ & 42.907 & 13.116 & $3.2 \mathrm{ML}$ & $26 / 10 / 2016$ & $5.9 \mathrm{Mw}$ & $0000: 18$ & 1.82 \\
\hline 21 & Central Italy & $26 / 10 / 2016$ & 42.889 & 13.128 & $3.0 \mathrm{ML}$ & $26 / 10 / 2016$ & $5.9 \mathrm{Mw}$ & $0000: 13$ & 2.22 \\
\hline 22 & Central Italy (S) & $26 / 10 / 2016$ & 42.872 & 13.141 & $2.2 \mathrm{ML}$ & $26 / 10 / 2016$ & $5.9 \mathrm{Mw}$ & $0000: 05$ & 4.23 \\
\hline 23 & Central Italy (F) & $26 / 10 / 2016$ & 42.879 & 13.157 & $4.3 \mathrm{ML}$ & $26 / 10 / 2016$ & $5.9 \mathrm{Mw}$ & 00 00:02 & 4.04 \\
\hline 24 & Central Italy (F) & $30 / 10 / 2016$ & 42.791 & 13.087 & $2.4 \mathrm{ML}$ & $30 / 10 / 2016$ & $6.5 \mathrm{Mw}$ & $0000: 36$ & 4.96 \\
\hline 25 & Central Italy & $30 / 10 / 2016$ & 43.062 & 13.068 & $2.1 \mathrm{ML}$ & $30 / 10 / 2016$ & $6.5 \mathrm{Mw}$ & $0000: 24$ & 25.81 \\
\hline 26 & Central Italy (S) & $30 / 10 / 2016$ & 42.927 & 13.029 & $2.0 \mathrm{ML}$ & $30 / 10 / 2016$ & $6.5 \mathrm{Mw}$ & 00 00:09 & 12.05 \\
\hline
\end{tabular}


As can be inferred, each second maximum (M) was preceded by shocks occurred with a maximum delay of about seven days and at a distance less than 85 $\mathrm{km}$. Most activation signals (red triangle) were activated a few minutes before the second maximum (M), while foreshocks (F) occurred approximately within seven days of the mainshock (M). FMA pattern completion occurred the moment when magnitude values dropped under the transition line, while the third maximum was formed within the dynamic range established by the transition line and the relevant parallel line drawn from the first maximum.

Earthquake pairs with comparable magnitude are included in the second category: one of them is often identifiable due to its greater magnitude compared to the other.

To recognise and classify large DEQ, from the U.S. Geological Survey (USGS) catalog we selected 1237 DEQ, whose magnitude was equal to or greater than 6 M, which occurred in various areas of the world from 1904 to 2017.

The criteria we used to select DEQ were: difference in magnitude between the two events, which should not be greater than 1.0 units and hypocentral depth that must not exceed $50 \mathrm{~km}$. For each pair of events we determined: time delay, distance in kilometers and difference in magnitude.

Our analyses highlighted that DEQ pattern has variances that allow us to group them into the types shown in Figure 7 and Figure 8 and are described below:

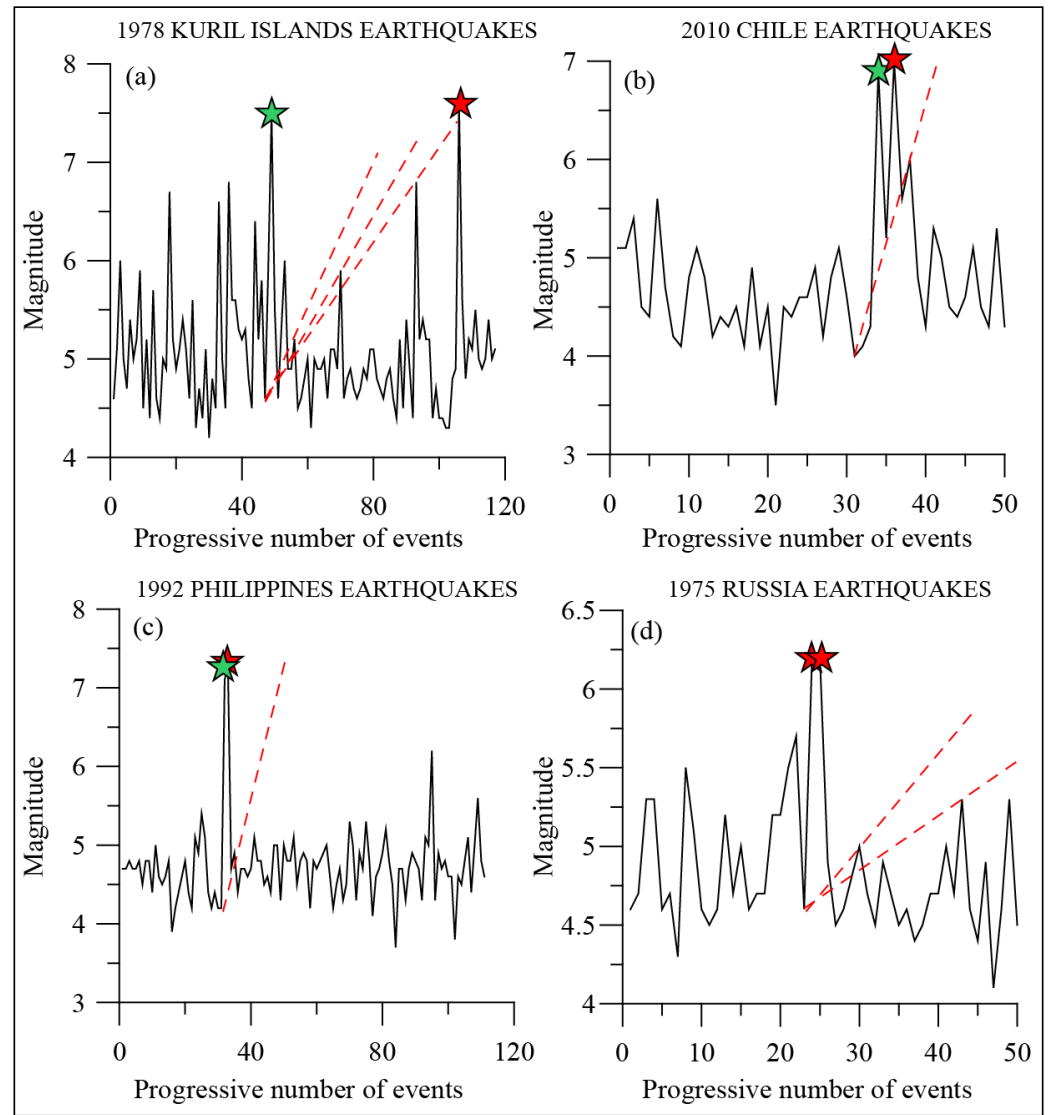

Figure 7. Types of DEQ patterns: (a) FM1, (b) FM2, (c) FM3, (d) MM. 


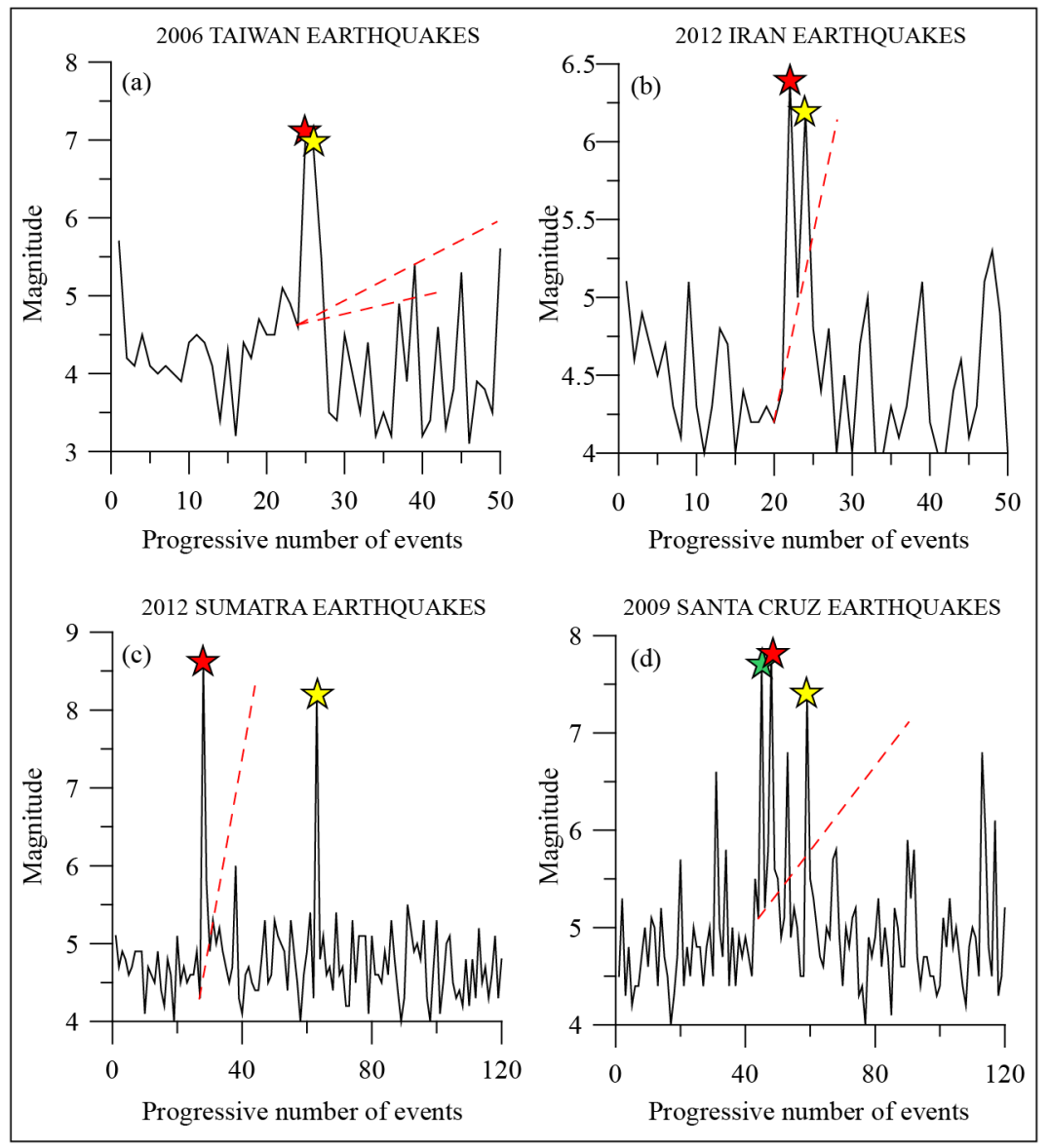

Figure 8. Types of DEQ patterns: (a) MA3, (b) MA2, (c) MA1, (d) Multiples.

a) DEQ consisting of one foreshock and one mainshock (FM1) separated by several shocks (Figure 7(a));

b) DEQ consisting of one foreshock and one mainshock (FM2) separated by a minimum (Figure 7(b));

c) DEQ consisting of one consecutive foreshock and mainshock (FM3) (Figure 7(c));

d) DEQ consisting of two consecutive mainshocks (MM) (Figure 7(d));

e) DEQ consisting of one consecutive mainshock and aftershock (MA3) (Figure 8(a));

f) DEQ consisting of one mainshock and one aftershock (MA2) separated by a minimum (Figure $8(\mathrm{~b})$ );

g) DEQ consisting of one mainshock and one aftershock (MA1) separated by several shocks (Figure 8(c));

h) DEQ multiples (Figure 8(d)).

In the Figure 7 and Figure 8, green, red and yellow stars, indicate the foreshock, mainshock and aftershock, respectively, while the dashed red line shows the transition line.

Figure 9 displays the correlations between distance, difference in magnitude and occurrence delays of DEQ analysed. 


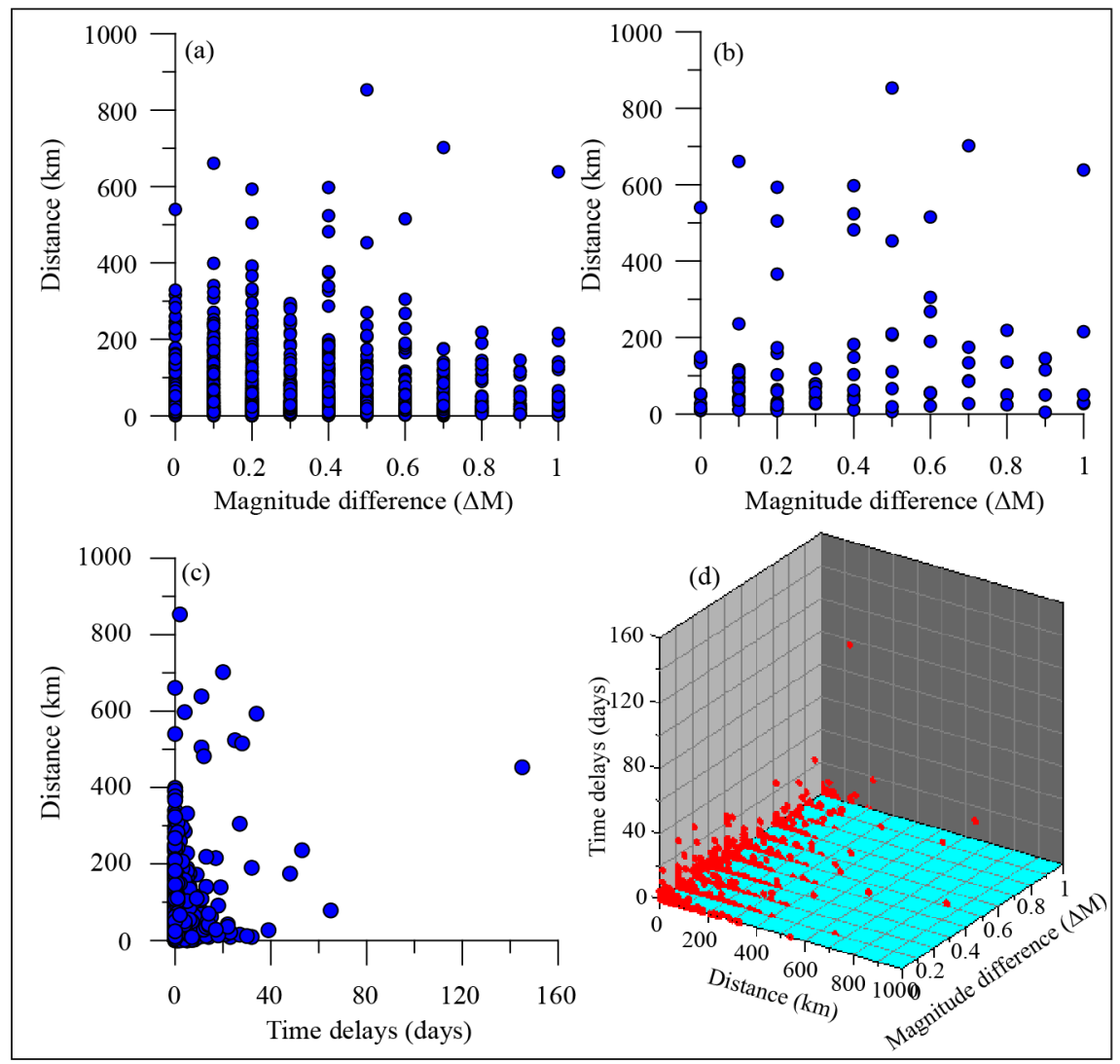

Figure 9. Correlations between DEQ's distance, magnitude difference and time delays. Figure 9(a) shows the correlation between distance and difference in magnitudes of DEQ with a magnitude $\geq 6.0$, while Figure 9 (b) concerns DEQ $\geq 7.0$. Figure 9 (c) shows the correlation between DEQ $(\geq 6.0 \mathrm{M})$ distance and occurrence delay and Figure $9(\mathrm{~d})$ shows the $3 \mathrm{D}$ correlation between DEQ occurrence delay, distance and difference in magnitudes.

As can be seen, many DEQ feature a fair close difference in magnitude with relatively short delays between two pair's events and occur close to each other.

Going into detail, $54.1 \%$ of DEQ shows a difference in magnitude equal to or less than 0.2 units, $83.6 \%$ less than 0.5 units and $93.4 \%$ less than 0.7 units. $50.9 \%$ is less than $30 \mathrm{~km}$ distant, $73.4 \%$ is less than $70 \mathrm{~km}$ distant and $81.6 \%$ is less than $100 \mathrm{~km}$ distant. $56.9 \%$ has a delay of less than one day, 81.1 less than three days and $95.7 \%$ less than 10 days. The greatest delay is 145 days.

The average distance is $61.3 \mathrm{~km}$, the average delay 1.93 days and the average magnitude difference is 0.29 units. $59.7 \%$ of DEQ whose difference in magnitude is 0.2 units has a delay of less than one day, $83.2 \%$ less than two days and $96.7 \%$ less than ten days. $50.1 \%$ is less than $30 \mathrm{~km}$ distant, $72.2 \%$ is less than $70 \mathrm{~km}$ and $80.0 \%$ is less than $100 \mathrm{~km}$.

$57.9 \%$ of DEQ with a difference in magnitude of 0.5 units has a delay of less than one day, $81.9 \%$ less than three days and $92.5 \%$ less than ten days. $50.9 \%$ is less than $30 \mathrm{~km}$ distant, $73.4 \%$ is less than $70 \mathrm{~km}$ and $81.5 \%$ is less than $100 \mathrm{~km}$ distant. 
Figure 10 reports percentage frequencies of DEQ occurrence delays and distances, in relation to $\leq 0.2$ and $\leq 0.5$ units differences in magnitude, respectively (Figures 10(a)-(d)).

Table 3 shows the difference in magnitude, time delay and distance between some DEQ, whose magnitude in equal to or higher than $7.0 \mathrm{M}$, occurred from 1904 to 2017 across the world.

Figure 11 shows analised DEQ's position and highlights that epicenters fall in the most seismic regions of the world-in some they are more frequent than in others. The region of the Solomon Islands and south-west Japan are two typical examples.

DEQ can be difficult to locate a priori, but in certain cases it is possible (patterns FM1, FM2, MA1 and MA2), to obtain the information that can be used to estimate their magnitude and occurrence time. Indeed, indications about when a DEQ may be formed, completed and the second event magnitude, are provided by reversal or extension points in relation to Fibonacci levels [21] [22].

For example, patterns FM2 and MA2 contain an upward or downward ABCD pattern (Figure 12) that most frequently has the following characteristics:

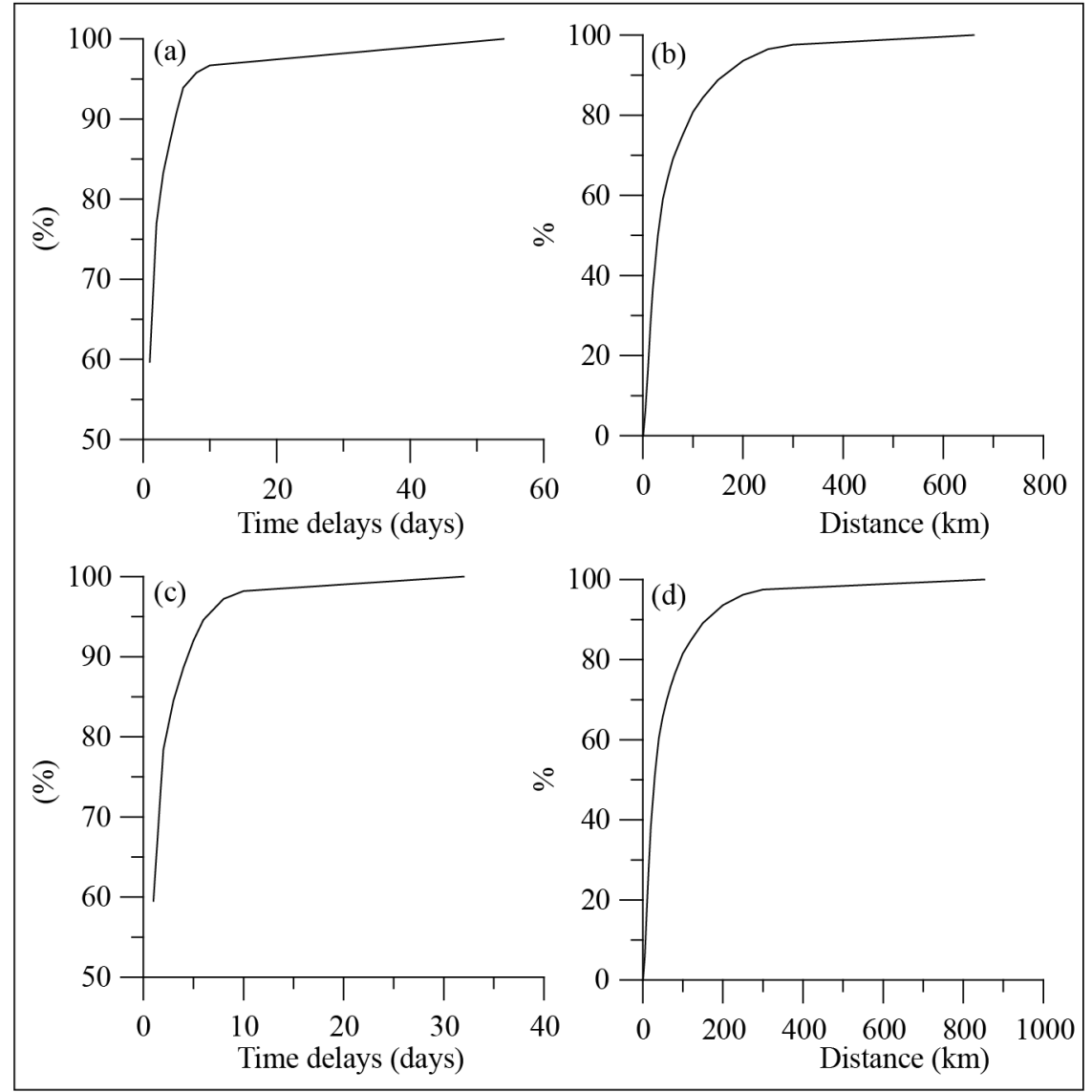

Figure 10. Figure 10(a) and Figure 10(b) show percentage frequencies of DEQ's occurrence delays and distances having a magnitude difference of $\leq 0.2$ units, while Figure 10(c) and Figure 10(d) show DEQ's occurrence delays and distance having a magnitude difference of $\leq 0.5$ units. 
Table 3. Earthquakes research parameters in USGS (number, earthquake, dates, latitude, longitude, depth and magnitude) catalog, difference in magnitude, delay between events and their distance.

\begin{tabular}{|c|c|c|c|c|c|c|c|c|c|}
\hline No & Earthquakes & Date & Lat & Long & $\begin{array}{c}\text { Depth } \\
(\mathrm{km})\end{array}$ & Magnitude & $\Delta \mathrm{M}$ & $\begin{array}{l}\text { Time Delays } \\
\text { (ddhh:mm) }\end{array}$ & $\begin{array}{c}\text { Distance } \\
(\mathrm{km})\end{array}$ \\
\hline 1 & Russia & $\begin{array}{l}25 / 06 / 1904 \\
25 / 06 / 1904\end{array}$ & $\begin{array}{l}52.864 \\
51.565\end{array}$ & $\begin{array}{l}160.445 \\
161.417\end{array}$ & $\begin{array}{l}15 \\
30\end{array}$ & $\begin{array}{l}7.5 \mathrm{Mw} \\
7.7 \mathrm{Mw}\end{array}$ & 0.2 & $0016: 15$ & 158.9 \\
\hline 2 & Mongolia & $\begin{array}{l}09 / 07 / 1905 \\
23 / 07 / 1905\end{array}$ & $\begin{array}{l}49.709 \\
49.369\end{array}$ & $\begin{array}{l}98.483 \\
96.610\end{array}$ & $\begin{array}{l}15 \\
15\end{array}$ & $\begin{array}{l}8.3 \mathrm{Mw} \\
8.3 \mathrm{Mw}\end{array}$ & 0.0 & $1317: 06$ & 140.3 \\
\hline 3 & Vanuatu & $\begin{array}{l}16 / 06 / 1910 \\
09 / 11 / 1910\end{array}$ & $\begin{array}{l}-19.572 \\
-16.289\end{array}$ & $\begin{array}{l}169.438 \\
166.904\end{array}$ & $\begin{array}{c}100 \\
20\end{array}$ & $\begin{array}{l}7.8 \mathrm{Mw} \\
7.3 \mathrm{Mw}\end{array}$ & 0.5 & $14523: 32$ & 452.9 \\
\hline 4 & Papua-Indonesia & $\begin{array}{l}13 / 01 / 1916 \\
13 / 01 / 1916\end{array}$ & $\begin{array}{l}-3.196 \\
-3.987\end{array}$ & $\begin{array}{l}135.731 \\
138.011\end{array}$ & $\begin{array}{l}25 \\
35\end{array}$ & $\begin{array}{l}7.1 \mathrm{Mw} \\
7.7 \mathrm{Mw}\end{array}$ & 0.6 & 00 02:02 & 267.9 \\
\hline 5 & Chile-Argentina & $\begin{array}{l}02 / 03 / 1919 \\
02 / 03 / 1919\end{array}$ & $\begin{array}{l}-43.800 \\
-43.109\end{array}$ & $\begin{array}{l}-78.319 \\
-71.695\end{array}$ & $\begin{array}{l}15 \\
15\end{array}$ & $\begin{array}{l}7.2 \mathrm{Mw} \\
7.2 \mathrm{Mw}\end{array}$ & 0.0 & $0008: 19$ & 540.0 \\
\hline 6 & Chile & $\begin{array}{l}07 / 11 / 1922 \\
11 / 11 / 1922\end{array}$ & $\begin{array}{l}-28.365 \\
-28.293\end{array}$ & $\begin{array}{c}-71.96 \\
-69.852\end{array}$ & $\begin{array}{l}25 \\
70\end{array}$ & $\begin{array}{l}7.0 \mathrm{Mw} \\
8.5 \mathrm{Mw}\end{array}$ & 0.5 & $0305: 32$ & 206.5 \\
\hline 7 & Japan & $\begin{array}{l}01 / 09 / 1923 \\
02 / 09 / 1923\end{array}$ & $\begin{array}{l}35.413 \\
35.007\end{array}$ & $\begin{array}{l}139.298 \\
139.926\end{array}$ & $\begin{array}{l}15 \\
15\end{array}$ & $\begin{array}{l}8.1 \mathrm{Mw} \\
7.8 \mathrm{Mw}\end{array}$ & 0.3 & $0023: 48$ & 72.7 \\
\hline 8 & China & $\begin{array}{l}03 / 07 / 1924 \\
11 / 07 / 1924\end{array}$ & $\begin{array}{l}36.983 \\
37.064\end{array}$ & $\begin{array}{l}84.164 \\
83.453\end{array}$ & $\begin{array}{l}10 \\
10\end{array}$ & $\begin{array}{l}\text { 7.2 Mw } \\
7.0 \mathrm{Mw}\end{array}$ & 0.2 & 08 15:04 & 63.8 \\
\hline 9 & Molucca Sea & $\begin{array}{l}03 / 05 / 1925 \\
03 / 06 / 1925\end{array}$ & $\begin{array}{l}1.190 \\
1.292\end{array}$ & $\begin{array}{l}126.01 \\
126.01\end{array}$ & $\begin{array}{l}15 \\
15\end{array}$ & $\begin{array}{l}7.1 \mathrm{Mw} \\
7.0 \mathrm{Mw}\end{array}$ & 0.1 & $3011: 12$ & 11.3 \\
\hline 10 & Bulgaria & $\begin{array}{l}14 / 04 / 1928 \\
18 / 04 / 1928\end{array}$ & $\begin{array}{l}42.329 \\
42.356\end{array}$ & $\begin{array}{l}25.717 \\
25.109\end{array}$ & $\begin{array}{l}10 \\
15\end{array}$ & $\begin{array}{l}7.1 \mathrm{Mw} \\
7.1 \mathrm{Mw}\end{array}$ & 0.0 & $0410: 22$ & 50.1 \\
\hline 11 & Mexico & $\begin{array}{l}17 / 06 / 1928 \\
04 / 08 / 1928 \\
09 / 10 / 1928\end{array}$ & $\begin{array}{l}16.182 \\
16.004 \\
16.190\end{array}$ & $\begin{array}{c}-96.585 \\
-98.209 \\
97.502\end{array}$ & $\begin{array}{l}20 \\
20 \\
25\end{array}$ & $\begin{array}{l}7.9 \mathrm{Mw} \\
7.2 \mathrm{Mw} \\
7.5 \mathrm{Mw}\end{array}$ & $\begin{array}{l}0.7 \\
0.3\end{array}$ & $\begin{array}{l}48 \text { 15:07 } \\
65 \text { 08:35 }\end{array}$ & $\begin{array}{c}174.6 \\
78.4\end{array}$ \\
\hline 12 & Maule & $\begin{array}{l}01 / 12 / 1928 \\
02 / 12 / 1928\end{array}$ & $\begin{array}{l}-35.155 \\
-35.685\end{array}$ & $\begin{array}{l}-72.105 \\
-72.812\end{array}$ & $\begin{array}{l}35 \\
30\end{array}$ & $\begin{array}{l}7.7 \mathrm{Mw} \\
7.0 \mathrm{Mw}\end{array}$ & 0.7 & $0024: 14$ & 87.1 \\
\hline 13 & Aleutian Islands & $\begin{array}{l}05 / 07 / 1929 \\
07 / 07 / 1929\end{array}$ & $\begin{array}{l}51.473 \\
51.474\end{array}$ & $\begin{array}{l}-178.152 \\
-177.771\end{array}$ & $\begin{array}{l}35 \\
35\end{array}$ & $\begin{array}{l}7.0 \mathrm{Mw} \\
7.3 \mathrm{Mw}\end{array}$ & 0.3 & 02 07:04 & 26.4 \\
\hline 14 & China & $\begin{array}{l}10 / 08 / 1931 \\
18 / 08 / 1931\end{array}$ & $\begin{array}{l}46.817 \\
47.264\end{array}$ & $\begin{array}{l}89.915 \\
89.859\end{array}$ & $\begin{array}{l}10 \\
10\end{array}$ & $\begin{array}{l}7.9 \mathrm{Mw} \\
7.1 \mathrm{Mw}\end{array}$ & 0.8 & $0717: 03$ & 49.9 \\
\hline 15 & Solomon Islands & $\begin{array}{l}03 / 10 / 1931 \\
03 / 10 / 1931 \\
03 / 10 / 1931 \\
10 / 10 / 1931\end{array}$ & $\begin{array}{c}-11.117 \\
-12.131 \\
-10.931 \\
-9.732\end{array}$ & $\begin{array}{l}161.110 \\
161.333 \\
161.414 \\
161.211\end{array}$ & $\begin{array}{l}15 \\
15 \\
15 \\
15\end{array}$ & $\begin{array}{l}7.9 \mathrm{Mw} \\
7.0 \mathrm{Mw} \\
7.0 \mathrm{Mw} \\
7.7 \mathrm{Mw}\end{array}$ & $\begin{array}{l}0.9 \\
0.0 \\
0.7\end{array}$ & $\begin{array}{l}0002: 05 \\
00: 01: 29 \\
0601: 32\end{array}$ & $\begin{array}{l}115.3 \\
133.7 \\
134.0\end{array}$ \\
\hline 16 & Mexico & $\begin{array}{l}03 / 06 / 1932 \\
18 / 06 / 1932 \\
22 / 06 / 1932\end{array}$ & $\begin{array}{l}19.786 \\
19.419 \\
19.373\end{array}$ & $\begin{array}{l}-103.784 \\
-103.907 \\
-104.224\end{array}$ & $\begin{array}{l}15 \\
15 \\
25\end{array}$ & $\begin{array}{l}8.1 \mathrm{Mw} \\
7.8 \mathrm{Mw} \\
7.7 \mathrm{Mw}\end{array}$ & $\begin{array}{l}0.3 \\
0.1\end{array}$ & $\begin{array}{l}1423: 36 \\
0402: 47\end{array}$ & $\begin{array}{l}42.8 \\
33.6\end{array}$ \\
\hline 17 & Santa Cruz Islands & $\begin{array}{l}18 / 07 / 1934 \\
21 / 07 / 1934\end{array}$ & $\begin{array}{l}-11.936 \\
-11.129\end{array}$ & $\begin{array}{l}166.977 \\
165.890\end{array}$ & $\begin{array}{l}10 \\
15\end{array}$ & $\begin{array}{l}7.7 \mathrm{Mw} \\
7.3 \mathrm{Mw}\end{array}$ & 0.4 & $0210: 38$ & 148.6 \\
\hline
\end{tabular}




\begin{tabular}{|c|c|c|c|c|c|c|c|c|c|}
\hline \multirow{2}{*}{18} & \multirow{2}{*}{ New Guinea } & $20 / 09 / 1935$ & -3.824 & 141.416 & 30 & 7.8 Mw & \multirow{2}{*}{0.8} & \multirow{2}{*}{$0003: 37$} & \multirow{2}{*}{135.9} \\
\hline & & $20 / 09 / 1935$ & -3.776 & 142.640 & 34 & 7.0 Mw & & & \\
\hline \multirow{2}{*}{19} & \multirow{2}{*}{ Japan } & $12 / 10 / 1935$ & 40.199 & 143.304 & 15 & 7.0 Mw & \multirow{2}{*}{0.1} & \multirow{2}{*}{05 07:27 } & \multirow{2}{*}{60.12} \\
\hline & & $18 / 10 / 1935$ & 40.235 & 144.011 & 15 & 7.1 Mw & & & \\
\hline \multirow{4}{*}{20} & \multirow{4}{*}{ Japan } & $05 / 11 / 1938$ & 36.966 & 142.090 & 35 & $7.8 \mathrm{Mw}$ & \multirow{4}{*}{$\begin{array}{l}0.1 \\
0.0 \\
0.1\end{array}$} & \multirow{4}{*}{$\begin{array}{l}00 \text { 02:07 } \\
00 \text { 22:03 } \\
00 \text { 12:45 }\end{array}$} & \multirow{4}{*}{$\begin{array}{l}25.1 \\
51.1 \\
43.1\end{array}$} \\
\hline & & $05 / 11 / 1938$ & 37.166 & 142.221 & 35 & $7.7 \mathrm{Mw}$ & & & \\
\hline & & $06 / 11 / 1938$ & 37.393 & 142.303 & 30 & $7.7 \mathrm{Mw}$ & & & \\
\hline & & $06 / 11 / 1938$ & 37.019 & 142.430 & 25 & 7.6 Mw & & & \\
\hline \multirow{2}{*}{21} & \multirow{2}{*}{ Costa Rica } & $05 / 12 / 1941$ & 8.396 & -83.457 & 20 & $7.3 \mathrm{Mw}$ & \multirow{2}{*}{0.3} & \multirow{2}{*}{$0100: 37$} & \multirow{2}{*}{118.6} \\
\hline & & $06 / 12 / 1941$ & 8.523 & -84.528 & 15 & 7.0 Mw & & & \\
\hline \multirow{2}{*}{22} & \multirow{2}{*}{ Dominican Republic } & 04/08/1946 & 19.083 & -69.248 & 15 & $7.5 \mathrm{Mw}$ & \multirow{2}{*}{0.5} & \multirow{2}{*}{$0319: 37$} & 663 \\
\hline & & $08 / 08 / 1946$ & 19.538 & -69.657 & 15 & 7.0 Mw & & & 00.3 \\
\hline 23 & Mariana Islands & $13 / 06 / 1947$ & 21.722 & 145.567 & 35 & $7.0 \mathrm{Mw}$ & 02 & $0511: 10$ & 172 \\
\hline 20 & 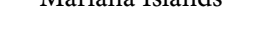 & $19 / 06 / 1947$ & 21.600 & 145.464 & 35 & 7.2 Mw & 0.2 & 0011.10 & 17.2 \\
\hline 24 & Tierra del Fuego & $17 / 12 / 1949$ & -53.923 & -69.596 & 10 & $7.7 \mathrm{Mw}$ & 04 & $00.08 \cdot 14$ & 104 \\
\hline 24 & 1 ierra del ruego & $17 / 12 / 1949$ & -53.911 & -69.753 & 10 & 7.3 Mw & 0.4 & $0008: 14$ & 10.4 \\
\hline 25 & Taiwan & $22 / 10 / 1951$ & 23.917 & 121.343 & 25 & $7.2 \mathrm{Mw}$ & 02 & $0002 \cdot 14$ & 166 \\
\hline 20 & Idiwdil & $22 / 10 / 1951$ & 23.775 & 121.393 & 20 & 7.0 Mw & 0.2 & 0002.14 & 10.0 \\
\hline 26 & Taiwan & $24 / 11 / 1951$ & 23.046 & 121.249 & 25 & $7.3 \mathrm{Mw}$ & 0.5 & $0000: 03$ & 6.2 \\
\hline & 1 diwdil & $24 / 11 / 1951$ & 23.092 & 121.214 & 30 & $7.8 \mathrm{Mw}$ & & & \\
\hline & & $11 / 03 / 1957$ & 52.691 & -169.191 & 35 & $7.1 \mathrm{Mw}$ & 0.1 & 00 04:57 & 660.8 \\
\hline & & $11 / 03 / 1957$ & 51.339 & -178.602 & 25 & 7.0 Mw & 0.1 & $0020: 50$ & 95.6 \\
\hline 27 & Aleutian Islands & $12 / 03 / 1957$ & 51.481 & -177.243 & 20 & $7.1 \mathrm{Mw}$ & 0.0 & 02 03:02 & 47.5 \\
\hline & & $14 / 03 / 1957$ & 51.196 & -176.733 & 25 & 7.1 Mw & 0.1 & $0111: 47$ & 115.8 \\
\hline & & $16 / 03 / 1957$ & 51.419 & -178.870 & 25 & $7.2 \mathrm{Mw}$ & & & \\
\hline 28 & Greece & $24 / 04 / 1957$ & 36.493 & 28.829 & 35 & 7.1 Mw & 0.2 & $0007 \cdot 15$ & 152 \\
\hline & Giecte th the & $25 / 04 / 1957$ & 36.405 & 28.699 & 35 & 7.3 Mw & & & \\
\hline 29 & Janan & $20 / 03 / 1960$ & 39.869 & 143.228 & 15 & $8.0 \mathrm{Mw}$ & 10 & $0207 \cdot 16$ & 271 \\
\hline 29 & Japdil & $23 / 03 / 1960$ & 39.635 & 143.316 & 15 & $7.0 \mathrm{Mw}$ & 1.0 & 0207.10 & 27.1 \\
\hline 30 & Chile & $21 / 05 / 1960$ & -37.824 & -73.353 & 25 & $8.1 \mathrm{Mw}$ & 10 & $0100 \cdot 28$ & 300 \\
\hline & & $22 / 05 / 1960$ & -37.775 & -73.017 & 25 & 7.1 Mw & 1.0 & 000.20 & 0.0 \\
\hline 31 & Japan & $16 / 01 / 1961$ & 36.121 & 141.758 & 30 & $7.2 \mathrm{Mw}$ & 02 & $0004: 52$ & 12.7 \\
\hline & Japdil & $16 / 01 / 1961$ & 36.226 & 141.815 & 30 & $7.0 \mathrm{Mw}$ & 0.2 & 0004.02 & \\
\hline 32 & Janan & $12 / 04 / 1962$ & 38.022 & 142.789 & 28 & $7.3 \mathrm{Mw}$ & 02 & 1105.06 & 505 \\
\hline 年 & Japant & $23 / 04 / 1962$ & 42.506 & 143.734 & 60 & 7.1 Mw & 0.2 & 1100.00 & 00 \\
\hline 33 & Indonesia & $15 / 04 / 1963$ & -0.975 & 128.07 & 30 & $7.1 \mathrm{Mw}$ & 0.0 & $0000: 26$ & 8.8 \\
\hline & & $16 / 04 / 1963$ & -1.050 & 128.043 & 30 & $7.1 \mathrm{Mw}$ & & & \\
\hline 34 & Santa Cruz Islands & $15 / 09 / 1963$ & -10.522 & 165.642 & 35 & 7.4 Mw & 02 & 0218.34 & 314 \\
\hline T & 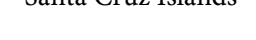 & $17 / 09 / 1963$ & -10.466 & 165.360 & 45 & 7.2 Mw & 0.2 & 0210.07 & J1.4 \\
\hline 35 & Kuril Islands & $13 / 10 / 1963$ & 44.872 & 149.483 & 35 & $8.5 \mathrm{Mw}$ & 0.7 & 06 19:36 & 85.5 \\
\hline & & 20/10/1963 & 44.726 & 150.547 & 28.2 & $7.8 \mathrm{Mw}$ & & & \\
\hline
\end{tabular}




\section{Continued}

\begin{tabular}{|c|c|c|c|c|c|c|c|c|c|}
\hline 36 & Kuril Islands & $\begin{array}{l}11 / 06 / 1965 \\
11 / 06 / 1965\end{array}$ & $\begin{array}{l}44.608 \\
44.578\end{array}$ & $\begin{array}{l}149.022 \\
148.699\end{array}$ & $\begin{array}{c}40.7 \\
58\end{array}$ & $\begin{array}{l}7.0 \mathrm{Mw} \\
7.2 \mathrm{Mw}\end{array}$ & 0.2 & $0000: 01$ & 25.8 \\
\hline 37 & Vanuatu & $\begin{array}{l}11 / 08 / 1965 \\
11 / 08 / 1965 \\
13 / 08 / 1965\end{array}$ & $\begin{array}{l}-15.449 \\
-15.861 \\
-15.871\end{array}$ & $\begin{array}{l}166.980 \\
167.092 \\
166.960\end{array}$ & $\begin{array}{l}25 \\
30 \\
25\end{array}$ & $\begin{array}{l}7.2 \mathrm{Mw} \\
7.6 \mathrm{Mw} \\
7.4 \mathrm{Mw}\end{array}$ & $\begin{array}{l}0.4 \\
0.2\end{array}$ & $\begin{array}{l}00 \text { 18:51 } \\
01 \text { 14:09 }\end{array}$ & $\begin{array}{l}47.4 \\
14.2\end{array}$ \\
\hline 38 & Santa Cruz Islands & $\begin{array}{l}31 / 12 / 1966 \\
31 / 12 / 1966\end{array}$ & $\begin{array}{l}-12.091 \\
-12.326\end{array}$ & $\begin{array}{l}166.552 \\
166.491\end{array}$ & $\begin{array}{l}55 \\
35\end{array}$ & $\begin{array}{l}7.8 \mathrm{Mw} \\
7.1 \mathrm{Mw}\end{array}$ & 0.7 & $0003: 52$ & 26.9 \\
\hline 39 & Japan & $\begin{array}{l}16 / 05 / 1968 \\
16 / 05 / 1968\end{array}$ & $\begin{array}{l}40.860 \\
41.430\end{array}$ & $\begin{array}{l}143.435 \\
142.864\end{array}$ & $\begin{array}{c}29.9 \\
25\end{array}$ & $\begin{array}{l}8.2 \mathrm{Mw} \\
7.9 \mathrm{Mw}\end{array}$ & 0.3 & $0009: 50$ & 79.4 \\
\hline 40 & Kuril Islands & $\begin{array}{l}11 / 08 / 1969 \\
14 / 08 / 1969\end{array}$ & $\begin{array}{l}43.599 \\
43.313\end{array}$ & $\begin{array}{l}147.385 \\
147.647\end{array}$ & $\begin{array}{c}25 \\
27.5\end{array}$ & $\begin{array}{l}7.5 \mathrm{Mw} \\
7.1 \mathrm{Mw}\end{array}$ & 0.4 & $0216: 52$ & 38.19 \\
\hline 41 & Papua New Guinea & $\begin{array}{l}14 / 07 / 1971 \\
26 / 07 / 1971\end{array}$ & $\begin{array}{l}-5.524 \\
-4.817\end{array}$ & $\begin{array}{l}153.850 \\
153.172\end{array}$ & $\begin{array}{l}40 \\
40\end{array}$ & $\begin{array}{l}8.0 \mathrm{Ms} \\
8.1 \mathrm{Ms}\end{array}$ & 0.1 & $1119: 12$ & 108.7 \\
\hline 42 & Russia & $\begin{array}{l}02 / 08 / 1971 \\
05 / 09 / 1971\end{array}$ & $\begin{array}{l}41.415 \\
46.505\end{array}$ & $\begin{array}{l}143.416 \\
141.199\end{array}$ & $\begin{array}{l}57.8 \\
18.1\end{array}$ & $\begin{array}{l}7.1 \mathrm{Mw} \\
7.3 \mathrm{Mw}\end{array}$ & 0.2 & $3411: 11$ & 593.1 \\
\hline 43 & Japan & $\begin{array}{l}17 / 06 / 1973 \\
24 / 06 / 1973\end{array}$ & $\begin{array}{l}43.233 \\
43.318\end{array}$ & $\begin{array}{l}145.785 \\
146.442\end{array}$ & $\begin{array}{l}48 \\
50\end{array}$ & $\begin{array}{l}7.7 \mathrm{Mw} \\
7.1 \mathrm{Mw}\end{array}$ & 0.6 & $0622: 48$ & 54.0 \\
\hline 44 & Solomon Islands & $\begin{array}{l}31 / 01 / 1974 \\
01 / 02 / 1974\end{array}$ & $\begin{array}{l}-7.461 \\
-7.383\end{array}$ & $\begin{array}{l}155.894 \\
155.575\end{array}$ & $\begin{array}{l}34 \\
40\end{array}$ & $\begin{array}{l}7.0 \mathrm{Ms} \\
7.1 \mathrm{Ms}\end{array}$ & 0.1 & $0003: 42$ & 36.2 \\
\hline 45 & Papua New Guinea & $\begin{array}{l}20 / 07 / 1975 \\
20 / 07 / 1975\end{array}$ & $\begin{array}{l}-6.590 \\
-7.104\end{array}$ & $\begin{array}{l}155.054 \\
155.152\end{array}$ & $\begin{array}{l}49 \\
44\end{array}$ & $\begin{array}{l}7.9 \mathrm{Ms} \\
7.7 \mathrm{Ms}\end{array}$ & 0.2 & $0005: 17$ & 58.2 \\
\hline 46 & Uzbekistan & $\begin{array}{l}08 / 04 / 1976 \\
17 / 05 / 1976\end{array}$ & $\begin{array}{l}40.311 \\
40.381\end{array}$ & $\begin{array}{l}63.773 \\
63.472\end{array}$ & $\begin{array}{l}33 \\
10\end{array}$ & $\begin{array}{l}7.0 \mathrm{Ms} \\
7.0 \mathrm{Ms}\end{array}$ & 0.0 & $3900: 18$ & 26.67 \\
\hline 47 & China & $\begin{array}{l}27 / 07 / 1976 \\
28 / 07 / 1976\end{array}$ & $\begin{array}{c}39.664 \\
39.57\end{array}$ & $\begin{array}{l}117.978 \\
117.978\end{array}$ & $\begin{array}{l}23 \\
26\end{array}$ & $\begin{array}{l}7.4 \mathrm{Mw} \\
7.4 \mathrm{Ms}\end{array}$ & 0.0 & $0015: 03$ & 10.4 \\
\hline 48 & Solomon Islands & $\begin{array}{l}20 / 04 / 1977 \\
20 / 04 / 1977 \\
20 / 04 / 1977\end{array}$ & $\begin{array}{l}-9.890 \\
-9.844 \\
-9.965\end{array}$ & $\begin{array}{l}160.348 \\
160.822 \\
160.731\end{array}$ & $\begin{array}{l}19 \\
33 \\
33\end{array}$ & $\begin{array}{l}7.5 \mathrm{Ms} \\
7.5 \mathrm{Ms} \\
7.5 \mathrm{Ms}\end{array}$ & $\begin{array}{l}0.0 \\
0.0\end{array}$ & $\begin{array}{l}00 \text { 00:07 } \\
00 \text { 04:35 }\end{array}$ & $\begin{array}{l}52.2 \\
16.7\end{array}$ \\
\hline 49 & Kuril Islands & $\begin{array}{l}23 / 03 / 1978 \\
24 / 03 / 1978\end{array}$ & $\begin{array}{l}44.932 \\
44.244\end{array}$ & $\begin{array}{l}148.439 \\
148.862\end{array}$ & $\begin{array}{l}33 \\
33\end{array}$ & $\begin{array}{l}7.5 \mathrm{Ms} \\
7.6 \mathrm{Ms}\end{array}$ & 0.1 & $0116: 32$ & 83.5 \\
\hline 50 & Mexico & $\begin{array}{l}07 / 06 / 1982 \\
07 / 06 / 1982\end{array}$ & $\begin{array}{l}16.610 \\
16.560\end{array}$ & $\begin{array}{l}-98.150 \\
-98.360\end{array}$ & $\begin{array}{l}40 \\
33\end{array}$ & $\begin{array}{l}7.2 \mathrm{Ms} \\
7.0 \mathrm{Ms}\end{array}$ & 0.2 & 00 03:20 & 23.0 \\
\hline 51 & Chile & $\begin{array}{l}03 / 03 / 1985 \\
04 / 03 / 1985 \\
09 / 04 / 1985\end{array}$ & $\begin{array}{l}-33.135 \\
-33.207 \\
-34.131\end{array}$ & $\begin{array}{l}-71.871 \\
-71.663 \\
-71.618\end{array}$ & $\begin{array}{c}33 \\
33 \\
37.8\end{array}$ & $\begin{array}{l}8.0 \mathrm{Mw} \\
7.4 \mathrm{Mw} \\
7.2 \mathrm{Mw}\end{array}$ & $\begin{array}{l}0.6 \\
0.2\end{array}$ & $\begin{array}{l}00 \text { 01:45 } \\
05 \text { 01:24 }\end{array}$ & $\begin{array}{c}20.9 \\
102.8\end{array}$ \\
\hline 52 & Papua New Guinea & $\begin{array}{l}10 / 05 / 1985 \\
03 / 07 / 1985\end{array}$ & $\begin{array}{l}-5.599 \\
-4.439\end{array}$ & $\begin{array}{l}151.045 \\
152.828\end{array}$ & $\begin{array}{c}26.7 \\
33\end{array}$ & $\begin{array}{l}7.2 \mathrm{Mw} \\
7.3 \mathrm{Mw}\end{array}$ & 0.1 & $5313: 00$ & 235.9 \\
\hline 53 & Afghanistan & $\begin{array}{l}29 / 07 / 1985 \\
23 / 08 / 1985\end{array}$ & $\begin{array}{l}36.190 \\
39.431\end{array}$ & $\begin{array}{l}70.896 \\
75.224\end{array}$ & $\begin{array}{c}98.7 \\
6.8\end{array}$ & $\begin{array}{l}7.4 \mathrm{Mw} \\
7.0 \mathrm{Mw}\end{array}$ & 0.4 & 25 04:47 & 523.7 \\
\hline 54 & Mexico & $\begin{array}{l}19 / 09 / 1985 \\
21 / 09 / 1985\end{array}$ & $\begin{array}{l}18.190 \\
17.802\end{array}$ & $\begin{array}{l}-102.533 \\
-101.647\end{array}$ & $\begin{array}{l}27.9 \\
30.8\end{array}$ & $\begin{array}{l}8.0 \mathrm{Mw} \\
7.6 \mathrm{Mw}\end{array}$ & 0.4 & $0112: 20$ & 103.2 \\
\hline
\end{tabular}




\section{Continued}

\begin{tabular}{|c|c|c|c|c|c|c|c|c|c|}
\hline 55 & Vanuatu & $\begin{array}{l}28 / 11 / 1985 \\
21 / 12 / 1985\end{array}$ & $\begin{array}{l}-13.987 \\
-13.966\end{array}$ & $\begin{array}{l}166.185 \\
166.516\end{array}$ & $\begin{array}{l}33 \\
43\end{array}$ & $\begin{array}{l}\text { 7.0 Mw } \\
\text { 7.1 Mw }\end{array}$ & 0.1 & $2221: 24$ & 35.8 \\
\hline 56 & Chile & $\begin{array}{l}05 / 03 / 1987 \\
05 / 03 / 1987\end{array}$ & $\begin{array}{l}-24.388 \\
-24.495\end{array}$ & $\begin{array}{l}-70.161 \\
-70.701\end{array}$ & $\begin{array}{l}62.3 \\
34.8\end{array}$ & $\begin{array}{l}\text { 7.6 Mw } \\
\text { 7.0 Mw }\end{array}$ & 0.6 & $0001: 38$ & 55.9 \\
\hline 57 & Papua New Guinea & $\begin{array}{l}12 / 10 / 1987 \\
16 / 10 / 1987\end{array}$ & $\begin{array}{l}-7.288 \\
-6.266\end{array}$ & $\begin{array}{c}154.371 \\
149.06\end{array}$ & $\begin{array}{l}24.7 \\
47.8\end{array}$ & $\begin{array}{l}\text { 7.0 Mw } \\
7.4 \mathrm{Mw}\end{array}$ & 0.4 & $0406: 51$ & 597.3 \\
\hline 58 & Fiji Islands & $\begin{array}{l}03 / 03 / 1990 \\
05 / 03 / 1990\end{array}$ & $\begin{array}{l}-22.122 \\
-18.318\end{array}$ & $\begin{array}{l}175.163 \\
168.063\end{array}$ & $\begin{array}{l}33.2 \\
20.7\end{array}$ & $\begin{array}{l}\text { 7.6 Mw } \\
7.1 \mathrm{Mw}\end{array}$ & 0.5 & $0204: 22$ & 852.9 \\
\hline 59 & Sudan & $\begin{array}{l}20 / 05 / 1990 \\
24 / 05 / 1990\end{array}$ & $\begin{array}{l}5.121 \\
5.358\end{array}$ & $\begin{array}{l}32.145 \\
31.848\end{array}$ & $\begin{array}{c}14.9 \\
16\end{array}$ & $\begin{array}{l}7.2 \mathrm{Mw} \\
7.1 \mathrm{Mw}\end{array}$ & 0.1 & $0417: 38$ & 42.1 \\
\hline 60 & Philippines & $\begin{array}{l}17 / 05 / 1992 \\
17 / 05 / 1992\end{array}$ & $\begin{array}{l}7.239 \\
7.191\end{array}$ & $\begin{array}{l}126.645 \\
126.762\end{array}$ & $\begin{array}{c}32.8 \\
33\end{array}$ & $\begin{array}{l}7.1 \mathrm{Mw} \\
7.3 \mathrm{Mw}\end{array}$ & 0.2 & $0000: 26$ & 13.9 \\
\hline 61 & Kuril Islands & $\begin{array}{l}04 / 10 / 1994 \\
09 / 10 / 1994\end{array}$ & $\begin{array}{l}43.773 \\
43.905\end{array}$ & $\begin{array}{l}147.321 \\
147.916\end{array}$ & $\begin{array}{l}14 \\
33\end{array}$ & $\begin{array}{l}8.3 \mathrm{Mw} \\
7.3 \mathrm{Mw}\end{array}$ & 1.0 & $0418: 33$ & 49.9 \\
\hline 62 & Japan & $\begin{array}{l}28 / 12 / 1994 \\
06 / 01 / 1995\end{array}$ & $\begin{array}{l}40.525 \\
40.246\end{array}$ & $\begin{array}{l}143.419 \\
142.175\end{array}$ & $\begin{array}{l}26.5 \\
26.9\end{array}$ & $\begin{array}{l}7.8 \mathrm{Mw} \\
7.7 \mathrm{Mw}\end{array}$ & 0.1 & $0910: 18$ & 109.8 \\
\hline 63 & Philippines & $\begin{array}{l}21 / 04 / 1995 \\
05 / 05 / 1995\end{array}$ & $\begin{array}{l}12.059 \\
12.626\end{array}$ & $\begin{array}{l}125.580 \\
125.297\end{array}$ & $\begin{array}{c}20.7 \\
16\end{array}$ & $\begin{array}{l}\text { 7.2 Mw } \\
7.1 \mathrm{Mw}\end{array}$ & 0.1 & $1403: 19$ & 70.1 \\
\hline 64 & Papua New Guinea & $\begin{array}{l}16 / 08 / 1995 \\
16 / 08 / 1995\end{array}$ & $\begin{array}{l}-5.799 \\
-5.771\end{array}$ & $\begin{array}{l}154.178 \\
154.347\end{array}$ & $\begin{array}{c}30.1 \\
33\end{array}$ & $\begin{array}{l}\text { 7.7 Mw } \\
\text { 7.2 Mw }\end{array}$ & 0.5 & $0012: 43$ & 18.9 \\
\hline 65 & Aleutian Islands & $\begin{array}{l}10 / 06 / 1996 \\
10 / 06 / 1996\end{array}$ & $\begin{array}{l}51.564 \\
51.478\end{array}$ & $\begin{array}{l}-177.632 \\
-176.847\end{array}$ & $\begin{array}{c}33 \\
26.3\end{array}$ & $\begin{array}{l}7.9 \mathrm{Mw} \\
7.3 \mathrm{Mw}\end{array}$ & 0.6 & $0011: 21$ & 55.1 \\
\hline 66 & Banda Sea & $\begin{array}{l}09 / 11 / 1998 \\
29 / 11 / 1998\end{array}$ & $\begin{array}{l}-6.92 \\
-2.071\end{array}$ & $\begin{array}{l}128.946 \\
124.891\end{array}$ & $\begin{array}{l}33 \\
33\end{array}$ & $\begin{array}{l}7.0 \mathrm{Mw} \\
7.7 \mathrm{Mw}\end{array}$ & 0.7 & $2008: 32$ & 701.9 \\
\hline 67 & Papua New Guinea & $\begin{array}{l}29 / 10 / 2000 \\
16 / 11 / 2000 \\
16 / 11 / 2000 \\
17 / 11 / 2000\end{array}$ & $\begin{array}{l}-4.766 \\
-3.980 \\
-5.233 \\
-5.496\end{array}$ & $\begin{array}{l}153.945 \\
152.169 \\
153.102 \\
151.781\end{array}$ & $\begin{array}{l}50 \\
33 \\
30 \\
33\end{array}$ & $\begin{array}{l}7.0 \mathrm{Mw} \\
8.0 \mathrm{Mw} \\
7.8 \mathrm{Mw} \\
7.8 \mathrm{Mw}\end{array}$ & $\begin{array}{l}1.0 \\
0.2 \\
0.0\end{array}$ & $\begin{array}{l}1720: 17 \\
0002: 48 \\
01 \quad 13: 19\end{array}$ & $\begin{array}{l}215.4 \\
173.5 \\
149.1\end{array}$ \\
\hline 68 & Peru & $\begin{array}{l}23 / 06 / 2001 \\
07 / 07 / 2001\end{array}$ & $\begin{array}{l}-16.265 \\
-17.543\end{array}$ & $\begin{array}{l}-73.641 \\
-72.077\end{array}$ & $\begin{array}{l}33 \\
33\end{array}$ & $\begin{array}{l}8.4 \mathrm{Mw} \\
7.6 \mathrm{Mw}\end{array}$ & 0.8 & 13 03:05 & 218.8 \\
\hline 69 & Japan & $\begin{array}{l}25 / 09 / 2003 \\
25 / 09 / 2003\end{array}$ & $\begin{array}{l}41.815 \\
41.774\end{array}$ & $\begin{array}{l}143.593 \\
143.593\end{array}$ & $\begin{array}{l}27 \\
33\end{array}$ & $\begin{array}{l}8.3 \mathrm{Mw} \\
7.4 \mathrm{Mw}\end{array}$ & 0.9 & $0001: 18$ & 4.5 \\
\hline 70 & Loyalty Islands & $\begin{array}{l}27 / 12 / 2003 \\
03 / 01 / 2004\end{array}$ & $\begin{array}{l}-22.015 \\
-22.015\end{array}$ & $\begin{array}{l}169.766 \\
169.683\end{array}$ & $\begin{array}{l}10 \\
22\end{array}$ & $\begin{array}{l}7.3 \mathrm{Mw} \\
7.1 \mathrm{Mw}\end{array}$ & 0.2 & $0700: 23$ & 8.5 \\
\hline 71 & Papua, Indonesia & $\begin{array}{l}05 / 02 / 2004 \\
07 / 02 / 2004\end{array}$ & $\begin{array}{l}-3.615 \\
-4.003\end{array}$ & $\begin{array}{l}135.538 \\
135.023\end{array}$ & $\begin{array}{c}16.6 \\
10\end{array}$ & $\begin{array}{l}\text { 7.0 Mw } \\
\text { 7.3 Mw }\end{array}$ & 0.3 & $0105: 37$ & 71.6 \\
\hline 72 & Sumatra, Indonesia & $\begin{array}{l}12 / 09 / 2007 \\
12 / 09 / 2007 \\
13 / 09 / 2007\end{array}$ & $\begin{array}{l}-4.438 \\
-2.625 \\
-2.130\end{array}$ & $\begin{array}{c}101.367 \\
100.841 \\
99.627\end{array}$ & $\begin{array}{l}34 \\
35 \\
22\end{array}$ & $\begin{array}{l}8.4 \mathrm{Mw} \\
7.9 \mathrm{Mw} \\
7.0 \mathrm{Mw}\end{array}$ & $\begin{array}{l}0.5 \\
0.9\end{array}$ & $\begin{array}{l}0012: 39 \\
00 \text { 03:46 }\end{array}$ & $\begin{array}{l}209.9 \\
145.7\end{array}$ \\
\hline 73 & Papua, Indonesia & $\begin{array}{l}03 / 01 / 2009 \\
03 / 01 / 2009\end{array}$ & $\begin{array}{l}-0.414 \\
-0.691\end{array}$ & $\begin{array}{l}132.885 \\
133.305\end{array}$ & $\begin{array}{l}17 \\
23\end{array}$ & $\begin{array}{l}7.7 \mathrm{Mw} \\
7.4 \mathrm{Mw}\end{array}$ & 0.3 & $0002: 50$ & 55.9 \\
\hline
\end{tabular}




\section{Continued}

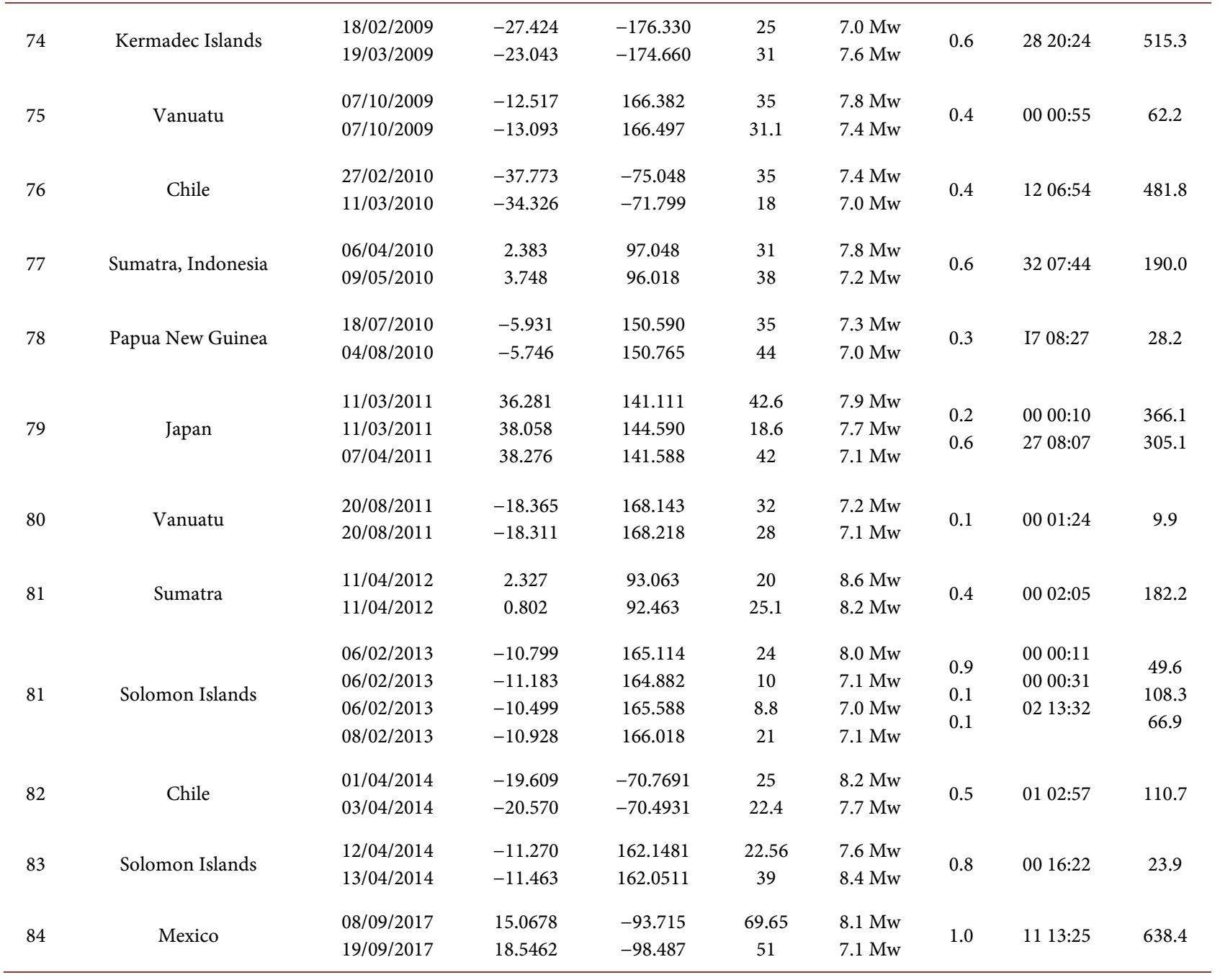

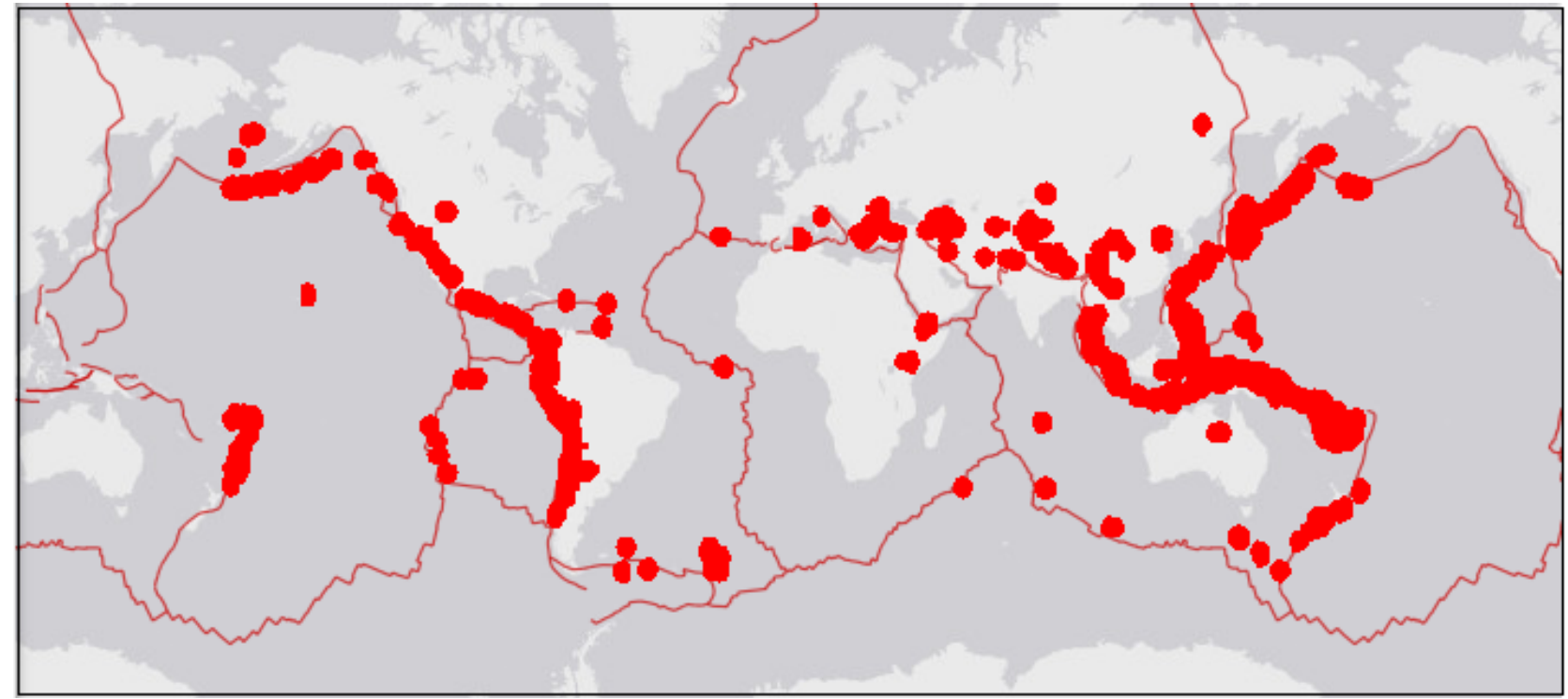

Figure 11. Location of DEQ whose magnitude is equal to or greater than 7.0 M occurred from 1904 to 2017 (base map USGS). 


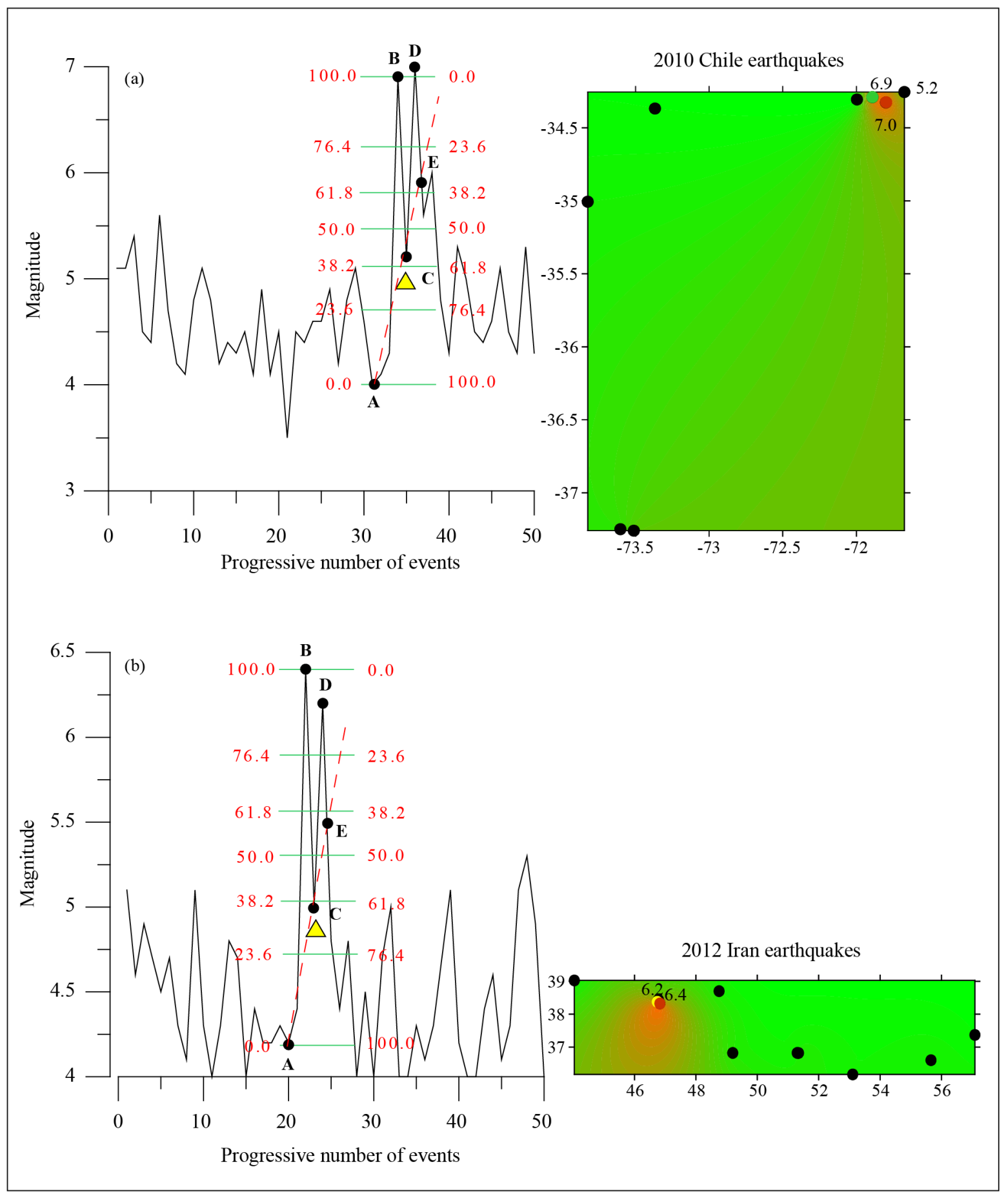

Figure 12. Fibonacci levels: (a) Chile Eartquakes 11/03/2010 (Time delays: 16 minutes), (b) Iran Eartquakes 11/08/2012 (Time delays: 11 minutes).

a) Segment BC is approximately $61.8 \%-76.4 \%$ of segment $\mathrm{AB}$;

b) Segment CD has approximately a \pm 0.5 units magnitude compared to point B.

The closer the $\mathrm{C}$ point to point B (segment BC less than 50\%) the greater is DEQ formation probability. Point E indicates the final magnitude values' drop 
below the transition line up to a magnitude value greater than the one indicated by point $\mathrm{C}$. Usually, this type of pattern takes a few minutes to complete, and we may assume that only one asperity breaks.

FM1 and MA1 patterns consisting of two EQ separated by multiple, minor shocks, have a high predictability ratio if we observe the "Butterfly" configuration (butterfly pattern) drawn by the shocks sequence between the two maxima. Figure 13 and Figure 14 report two examples of FM1 and MA1 patterns, respectively, where $A B C D$ pattern is upwards, and segment $B C$ is approximately greater than $0.764 \%$ of segment $A B$ (Figure 13(a) and Figure 14(a)).

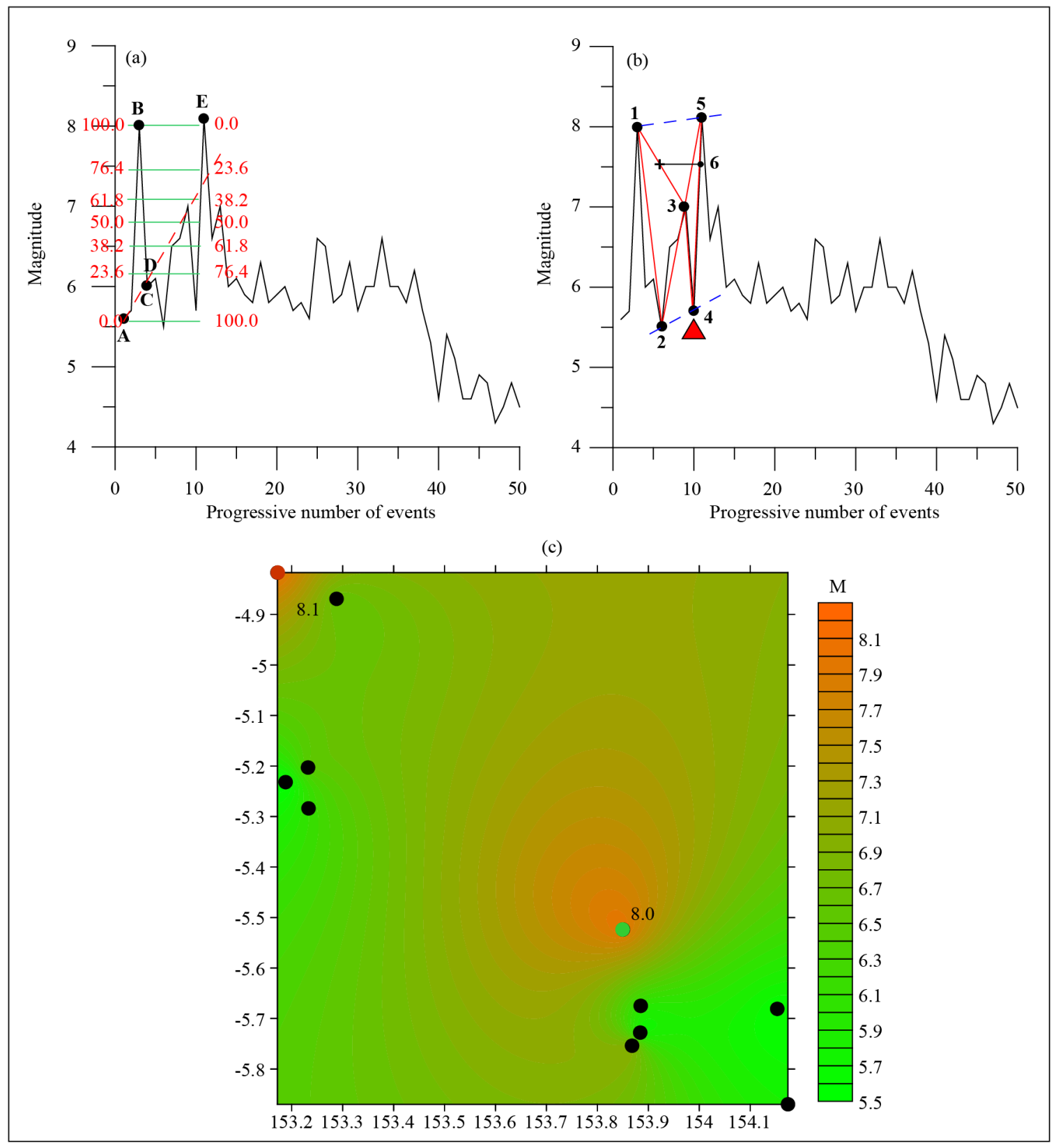

Figure 13. New Guinea earthquakes of 14/07/1971 and 26/07/1971 (Time delays: 12 days 19 hours 12 minutes). In Figure 13(c), red circle indicates the mainshock, while green circle the foreshock. Black circles indicate low magnitude shocks. 


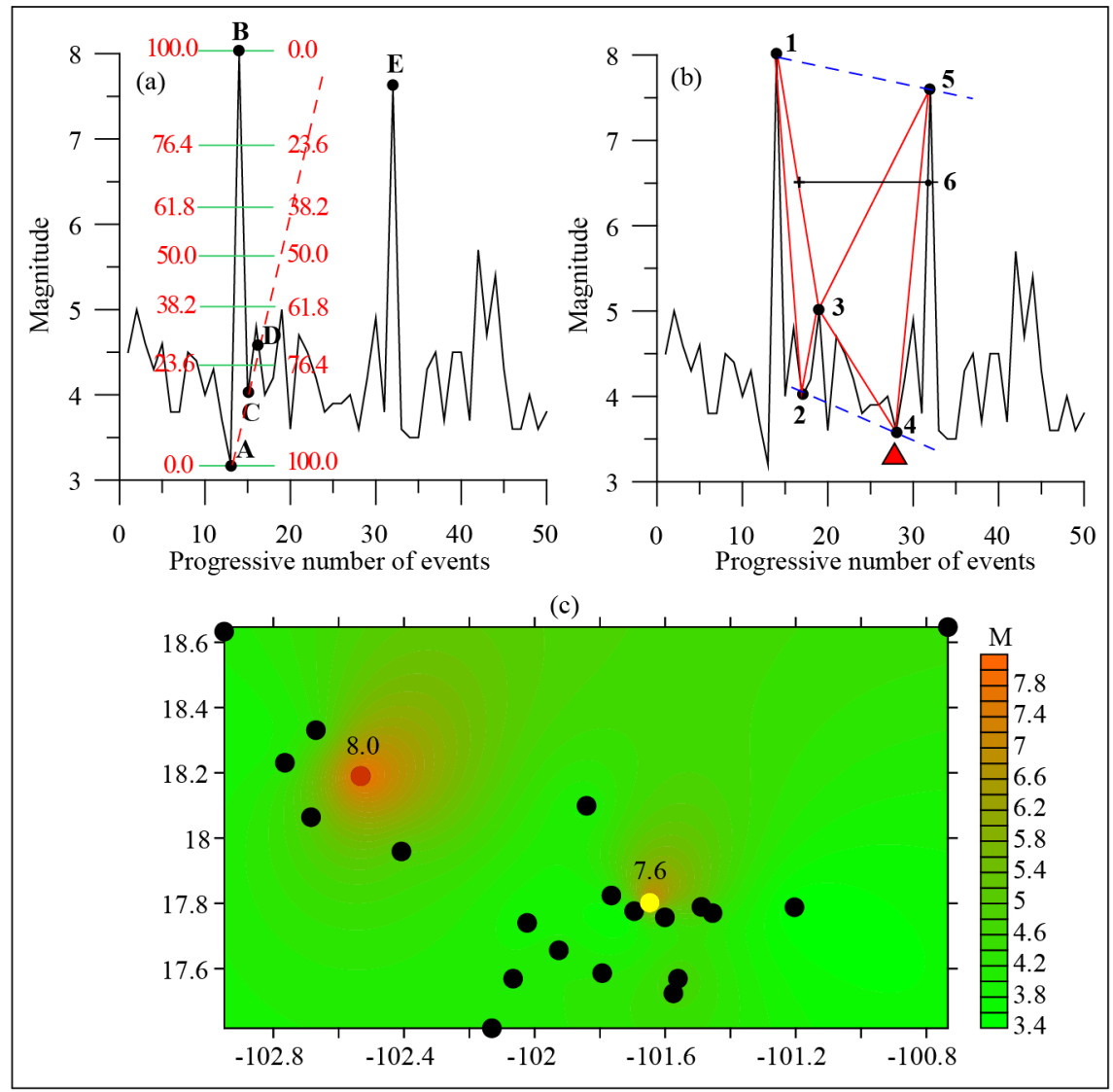

Figure 14. Mexico earthquakes of 19/09/1985 and 21/09/1985 (Time delays 1 day 12 hours 20 minutes). In Figure 14(c), the red circle indicates the mainshock, while the yellow circle the aftershock. Black circles indicate low magnitude shocks.

"Butterfly" configuration (Figure 13(b) and Figure 14(b)) is better described by the connection of points $1,2,3,4$ and 5 that represent, respectively:

- Points 1 and 5, the first and the second earthquake;

- Point 3, the second relative maximum that forms after point 1 ;

- Points 2 and 4 the first and the second minima that form before and after point 3.

To define the configuration, we must connect the first maximum (point 1) with the first minimum (point 2) and then the latter with the second maximum (point 3 ) that confirms the point 2 previously identified. From point 3 the magnitude values begin to drop until they form the second minimum (point 4) and, finally, the point 5 will complete the "butterfly" pattern. It is possible to draw multiple, temporary "Butterfly" patterns between two separate EQ with the consequent formation of multiple points 3 of increasing magnitude. In this case, for the pattern construction we should consider the last point 3 formed.

Once defined, the "Butterfly" pattern allows obtaining the following information:

a) The higher the point 3 (above $50 \%$ level), the greater the probability that points 2 and 4 are increasing and the point 5 is greater in magnitude compared 
to point 1 (point 1 is a foreshock, while point 5 is a mainshock);

b) The lower the point 3 (below 50\% level), the greater the probability that points 2 and 4 are decreasing and the point 5 is smaller in magnitude compared to point 1 (point 1 is a mainshock, while the point 5 is an aftershock);

c) Usually, between points 1 and 2 and 3 and 4, the minimum magnitude values have a decreasing trend, while trend is increasing between points 2 and 3 and 4 and 5;

d) The minimum magnitude value of the second maximum is given by point 1 and 3 average magnitude values (point 6);

e) The second maximum highest magnitude value is empirically calculated by adding to (if points 2 and 4 are upwards) or subtracting from (if points 2 and 4 are downwards) the first maximum magnitude value, the magnitude difference between points 2 and 4;

f) The activation signal coincides with point 4 (red triangle);

g) Usually, the area formed by points 1, 2 and 3 (pattern's left sector) is larger if minima 2 and 4 are upward, while it is smaller if the contrary happens;

h) Second DEQ earthquake is more frequently of "Flash Earthquake" type (i.e., foreshocks do not precede it);

i) Often some minor shocks that occur before DEQ are close to their epicenters (Figure 13(c) and Figure 14(c)).

This pattern takes longer to complete (from a few hours to several days) and, given its asymmetry highlighted by point 3 position, we may assume the breaking of many asperities, faults or different rocks.

The second maximum's magnitude values range within the "Butterfly" pattern can be estimated by the following procedure as well:

$$
\begin{aligned}
& M_{1}=P_{M}+0.618 \cdot\left(M_{P 1}-M_{P 2}\right) \\
& M_{2}=P_{M}+0.382 \cdot\left(M_{P 1}-M_{P 2}\right)
\end{aligned}
$$

where,

$M_{1}=$ magnitude value of the range upper limit;

$M_{2}=$ magnitude value of the range lower limit;

$$
P_{M}=\left(M_{P 1}+M_{P 2}+M_{P 3}\right) / 3
$$

$M_{P 1}, M_{P 2}, M_{P 3}=$ magnitude values of points 1, 2 and 3 in the pattern;

0.382 and 0.618 are Fibonacci levels statistically achieved by the magnitude values of the second event in the pattern.

The procedure we present here can also be used to assess the magnitude value of the second earthquake (point D) in patterns FM2 and MA2 by considering the following points:

$$
\begin{aligned}
& M_{P 1}=\text { point } \mathrm{B} \text { magnitude value; } \\
& M_{P 2}=\text { point } \mathrm{A} \text { magnitude value; } \\
& M_{P 3}=\text { point } \mathrm{C} \text { magnitude value. }
\end{aligned}
$$

Figure 15 shows an example of MEQ (Multiples Earthquakes), in which the second EQ happened with a delay (time delays) of 2 hours 48 minutes and at a 

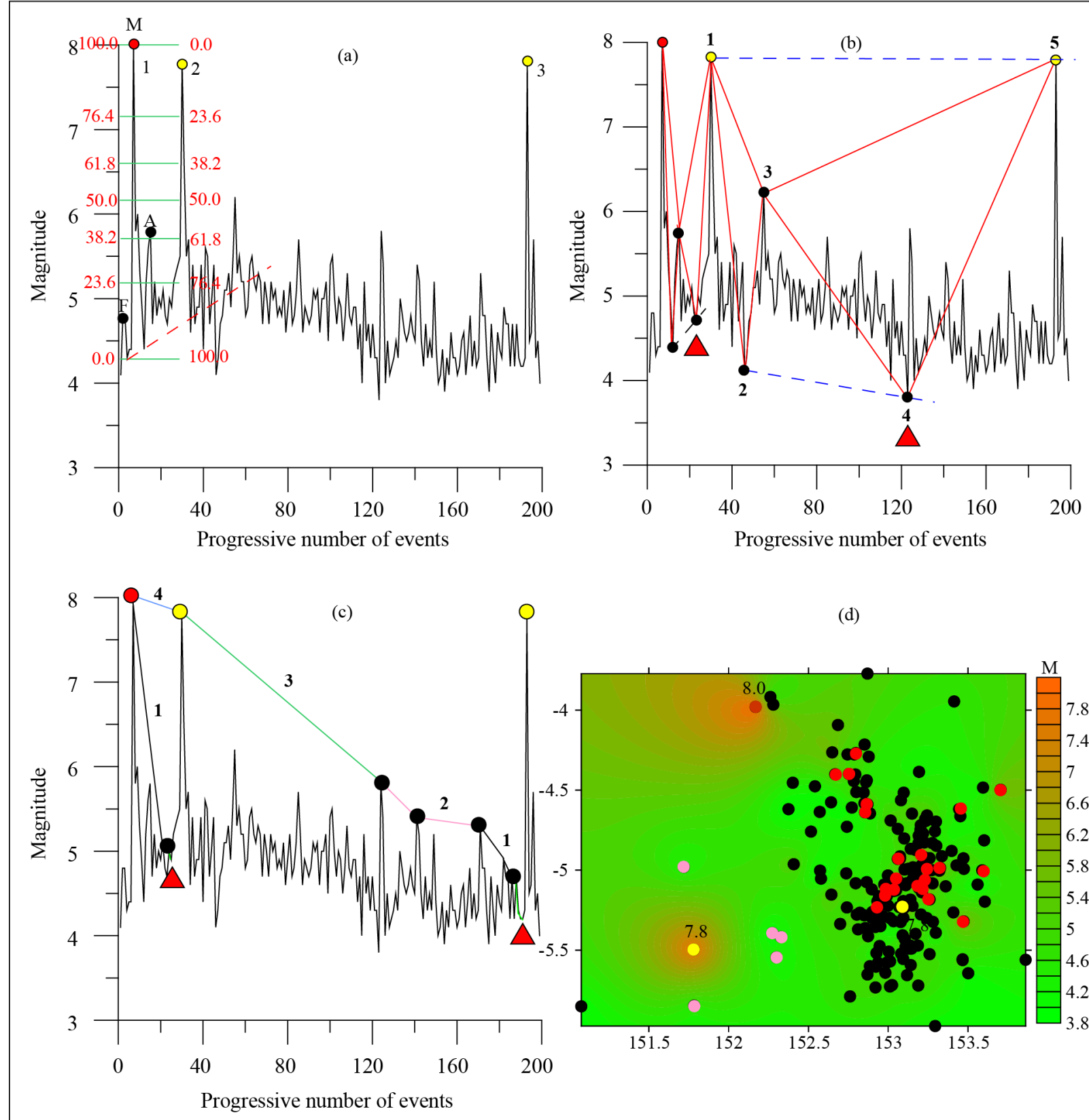

(d)

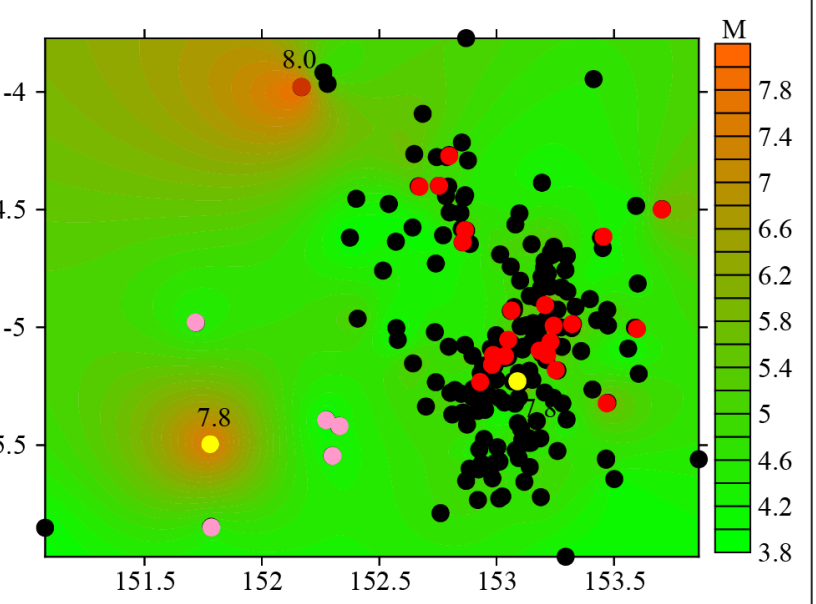

Figure 15. New Guinea earthquakes of 16/11/2000 and 17/11/2000.

$173.5 \mathrm{~km}$ distance from the first. The third EQ occurred with 1 days 13 hours 19 minutes delay and at a 149.1 distance $\mathrm{km}$ from the second.

The longer delay observed in third EQ compared to the first is also highlighted by the higher number of shocks occurred. After the first seismic event, we observe a decrease in magnitude values up to almost 100\% (Figure 15(a)) and a final rise pretty close to the first earthquake $(-0.2$ units). In (Figure 15(b)), we notice that stronger earthquakes are separated by several shocks forming asymmetric butterfly patterns. Figure 15 (c) shows branched structures [16] and activation signals of energy release phases preceding the second and 
third EQ (signals are delayed compared to those provided by the "Butterfly" pattern). Figure 15(d) reports the epicenters of seismic events occurred in the first 8.0 Mw magnitude and the last 7.8 Mw magnitude earthquakes.

After the initial 8.0 Mw magnitude earthquake, we observe a SE-oriented migration of subsequent shocks epicenters (red circles), where we see a higher concentration of seismicity in a region surrounding the second 7.8 Mw magnitude EQ (yellow circle). During the aftershock phase, we note that some epicenters (magenta-colored) are arranged around the epicenter of the third 7.8 Mw event.

Figure 16 shows another example of MEQ consisting of two DEQ: the first consists of two earthquakes having $6.9 \mathrm{Mw}$ and $6.8 \mathrm{Mw}$ magnitude, respectively, while the second consists of consecutive earthquakes whose magnitudes are 6.8 $\mathrm{Mw}$ and 7.2 Mw. After the first 6.9 Mw event (Figure 16(a)), we observe a decrease in magnitude values up to nearly $61.8 \%$ (point $C$ ) and a first rise (point $\mathrm{D}$ ) almost close to first earthquake ( -0.1 units). The delay (time delays) between the first and second earthquake was 21 minutes and the distance was $13.84 \mathrm{~km}$. The third 7.2 Mw earthquake (point D1) occurred 4 minutes after the second, at a distance of $15 \mathrm{~km}$.

Figure 16(b) shows that events' epicenters (black circles) between point $\mathrm{F}$ and point $\mathrm{A}$ (Figure 16(a)) and the minimum $\mathrm{C}$ having a $5.2 \mathrm{Mw}$ magnitude (yellow circle), are close to the strongest EQ's future epicenters (red and green circles)

This information suggests that the most complex DEQ e MEQ patterns can be related to multiple asperities having different sizes and thickness, located along the same or adjacent faults.

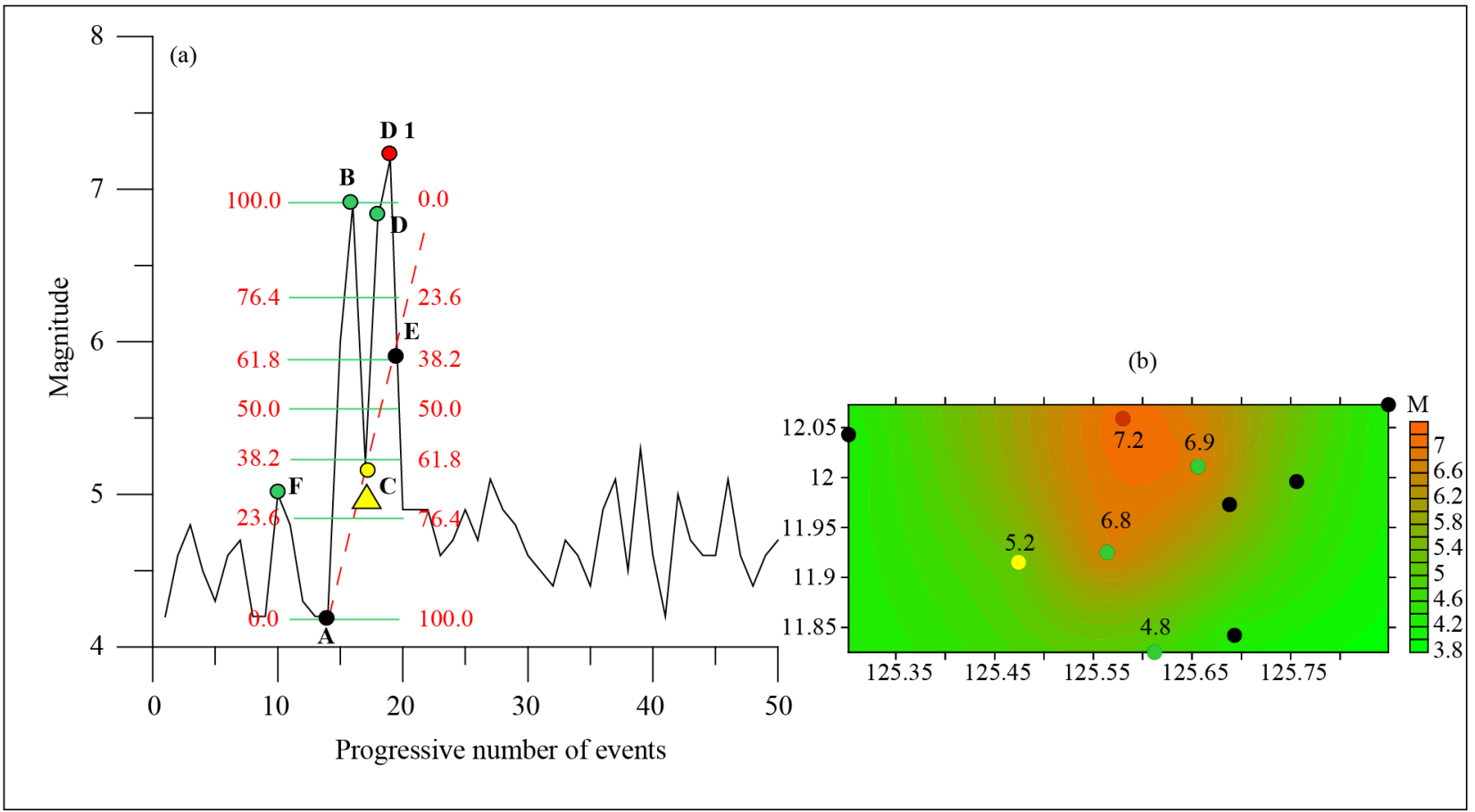

Figure 16. Philippines earthquakes of 21/04/1995. 
In fact, along the faults with large asperities having similar thickness, the breaking of one of them leads to an increasing stress in the adjacent ones by triggering their breaking and hence the formation of distinct, but similar earthquakes pairs [3].

\section{Conclusions}

In this paper, we used a graphic and statistical approach to classify double and multiple earthquakes and identify the activation signals that allow collecting information about the time of the second earthquake, whose magnitude is comparable to the first, may happen.

The observations made on magnitude values fluctuations over time, underline that FMA pattern allows identifying the energy release phase closure as well as the mainshock compared to that of other major earthquakes in the pattern.

In some seismic sequences, FMA pattern consists of two or more earthquakes of magnitude comparable: we may classify them depending on the number of lower magnitude shocks that separate them.

The detailed analysis of 1237 DEQ occurred across the world show that, as a rule, an earthquake within the FMA pattern can trigger a second large event close in time and space.

The results we obtained show that a 0.5 units magnitude difference, a spatial separation not exceeding $100 \mathrm{~km}$ and 10 days time separation are DEQ's most common characteristics.

In different types of DEQ, we noticed that as the number of shocks between the first and the second event increases, even the distance between the events basically increases (the earthquake pairs are most likely associated with the breaking of several asperities along the same or adjacent faults), while in the absence of a shock or a few shocks between DEQ, the distance between events essentially decreases (earthquake pairs are most likely associated with repeated breaking of the same asperity or fault).

Besides, in DEQ separated by several lower magnitude shocks, a "Butterfly" pattern is formed, which allows obtaining early information on the second earthquake pairs' magnitude and when this will happen.

Patterns consisting of more than two earthquakes with comparable magnitude (MEQ) differ from basic FMA pattern, and probably they are formed in certain complex tectonic areas where crustal asperieties having different sizes and thickness, may be several.

Usually, these patterns result from a mainshock and two or more foreshocks/aftershocks having similar magnitude although differently spaced in time and space.

Large earthquakes with comparable magnitude are not rare and in some areas could represent an underestimated risk.

In our approach, we believe that the position of the trigger point immediately generated after the first earthquake in some types of $\mathrm{DEQ}$, is the most hazardous 
point and, therefore, its identification is crucial to reduce the risks that rescue teams are exposed to if the following earthquake features a comparable seismic energy.

\section{References}

[1] Schwartz, S.Y., Lay, T. and Ruff, L.J. (1989) Source Process of the Great 1971 Solomon Islands Doublet. Physics of the Earth and Planetary Interiors, 56, 294-310. https://websites.pmc.ucsc.edu/ thorne/TL.pdfs/SLR_Solomon_PEPI1989.pdf https://doi.org/10.1016/0031-9201(89)90164-7

[2] Xu, Z. and Schwartz, S.Y. (1993) Large Earthquake Doublets and Fault Plane Heterogeneity in the Northern Solomon Islands Subduction Zone. Pure and Applied Geophysics, 140, 365-390. https://link.springer.com/article/10.1007/BF00879412 https://doi.org/10.1007/BF00879412

[3] Lay, T. and Kanamori, H.K. (1980) Earthquake Doublets in the Solomon Islands. Physics of the Earth and Planetary Interiors, 21, 283-304. https://websites.pmc.ucsc.edu/ thorne/TL.pdfs/LK_solomon_pepi80.pdf

[4] Astiz, L. and Kanamori, H. (1984) An Earthquake Doublet in Ometepec, Guerrero, Mexico. Physics of the Earth and Planetary Interiors, 34, 24-45.

http://gps-prod-storage.cloud.caltech.edu.s3.amazonaws.com/people_personal_asse ts/kanamori/HKpepi84.pdf https://doi.org/10.1016/0031-9201(84)90082-7

[5] King, G.C.P., Stein, R.S. and Lin, J. (1994) Static stress Changes and the Triggering of Earthquakes. Bulletin of the Seismological Society of America, 84, 935-955. http://www.ipgp.fr/ king/Geoffrey_King/Publications_files/King1994\%20BSSA.pdf

[6] Stein, R.S., King, G.C. and Lin, J. (1994) Change in Failure Stress on the Southern San Andreas Fault System Caused by the 1992 Magnitude = 7.4 Landers Earthquake. Science, 258, 1328-1332.

https://www.researchgate.net/profile/Geoffrey_King/publication/6062900_Change_ in_Failure_Stress_on_the_Southern_San_Andreas_Fault_System_Caused_by_the_1 992_Magnitude_74_Landers_Earthquake/links/02bfe51151641de77f000000.pdf https://doi.org/10.1126/science.258.5086.1328

[7] Deng, J. and Sykes, L.R. (1997) Evolution of the Stress Field in Southern California and the Triggering of Moderate-Size Earthquakes: A 200-Year Perspective. Journal of Geophysical Research, 102, 9859-9886.

http://onlinelibrary.wiley.com/doi/10.1029/96JB03897/full https://doi.org/10.1029/96JB03897

[8] Gomberg, J., Beeler, N.M., Blanpied, M.L. and Bodin, P. (1998) Earthquake Triggering by Transient and Static Deformations. Journal of Geophysical Research, 103, 24411-24426. https://doi.org/10.1029/98JB01125

[9] Stein, R.S. (1999) The Role of Stress Transfer in Earthquake Occurrence. Nature, 402, 605-609. https://doi.org/10.1038/45144 http://basin.earth.ncu.edu.tw/download/courses/seminar_MSc/2011/1229-1_02.pdf

[10] Ammon, C.J., Kanamori, H. and Lay, T. (2008) A Great Earthquake Doublet and Seismic Stress Transfer Cycle in the Central Kuril Islands. Nature, 451, 561-565.

https://www.ncbi.nlm.nih.gov/pubmed/18235499 https://doi.org/10.1038/nature06521

[11] Ruff, L. (1992) Asperity Distributions and Large Earthquake Occurrence in Subduction Zones. Tectonophysics, 211, 61-83.

http://citeseerx.ist.psu.edu/viewdoc/download?doi=10.1.1.961.280\&rep=rep1\&type $=\mathrm{pdf}$ 
[12] Kase, Y. and Kuge, K. (2001) Rupture Propagation beyond Fault Discontinuities: Significance of Fault Strike and Location. Geophysical Journal International, 147, 330-342. https://doi.org/10.1046/j.1365-246X.2001.00533.x

https://academic.oup.com/gji/article/147/2/330/718353/Rupture-propagation-beyon d-fault-discontinuities

[13] Duan, B. and Oglesby, D.D. (2006) Heterogeneous Fault Stresses from Previous Earthquakes and the Effect on Dynamic of Parallel Strike-Slip Faults. Journal of Geophysical Research, 111, B05309. https://doi.org/10.1029/2005JB004138 http://people.tamu.edu/ BDUAN/Duan_2005JB004138.pdf

[14] Felzer, K.R., Abercrombie, R.E. and Ekström, G. (2004) A Common Origin of Aftershocks, Foreshocks, and Multiplets. Bulletin of the Seismological Society of America, 94, 88-98.

http://citeseerx.ist.psu.edu/viewdoc/download?doi=10.1.1.574.3527\&rep=rep1\&type $=\mathrm{pdf}$

[15] Riga, G. and Balocchi, P. (2016) Short-Term Earthquake Forecast with the Seismic Sequence Hierarchization Method. Open Journal of Earthquake Research, 5, 79-96. https://doi.org/10.4236/ojer.2016.52006

[16] Riga, G. and Balocchi, P. (2016) How to Identify Foreshocks in Seismic Sequences to Predict Strong Earthquakes. Open Journal of Earthquake Research, 5, 55-71. http://file.scirp.org/pdf/OJER_2017022716403939.pdf https://doi.org/10.4236/ojer.2017.61003

[17] Riga, G. and Balocchi, P. (2016) How to Predict Earthquakes with Microsequences and Reversed Phase Repetitive Patterns. Open Journal of Earthquake Research, 5, 153-164. http://file.scirp.org/pdf/OJER_2016070913465665.pdf https://doi.org/10.4236/ojer.2016.53012

[18] Riga, G. and Balocchi, P. (2016) Seismic Sequence Structure and Earthquakes Triggering Patterns. Open Journal of Earthquake Research, 5, 20-34. https://doi.org/10.4236/ojer.2016.51003

[19] INGV (2017) Centro Nazionale Terremoti: Personalizza Ricerca. http://cnt.rm.ingv.it/search?starttime $=2018-01-20+00 \% 3 \mathrm{~A} 00 \% 3 \mathrm{~A} 00$ \&endtime $=201$ 8-01-27+23\%3A59\%3A59\&last_nd=7\&minmag=2\&maxmag $=10 \&$ mindepth $=-10 \&$ maxdepth $=1000 \&$ minlat $=-90 \&$ maxlat $=90 \&$ minlon $=-180 \&$ maxlon $=180 \&$ minversion $=100 \&$ limit $=30$ \&orderby $=$ ot - desc $\&$ tdmt_flag $=-1$ \&lat $=0 \&$ lon $=0 \&$ maxradiuskm $=-1 \&$ wheretype $=$ area

[20] U.S. Geological Survey (USGS). Search Earthquake Archives. http://earthquake.usgs.gov/earthquakes/search/

[21] Boroden, C. (2008) Fibonacci Trading. How to Master the Time and Price Advantage. McGraw-Hill Finance \& Investing, New York.

https://fx-arabia.com/vb/uploaded/178_01279135087.pdf

[22] Kumar, R. (2014) Magic of Fibonacci Sequence in Prediction of Stock Behavior. International Journal of Computer Applications, 93, 36-40. https://pdfs.semanticscholar.org/a2e3/e5a882f88f521590cb436ecdf271eb1ac294.pdf https://doi.org/10.5120/16262-5926 